\title{
Influence of convective heat transfer modelling in CFD simulations of upward flame spread
}

\author{
Georgios Maragkos ${ }^{\mathrm{a}, *}$, Davood Zeinali ${ }^{\mathrm{b}}$, Bart Merci $^{\mathrm{a}}$ \\ ${ }^{a}$ Department of Structural Engineering and Building Materials, Ghent University, St. \\ Pietersnieuwstraat 41, B-9000 Ghent, Belgium \\ ${ }^{b}$ Université de Lorraine, CNRS, LEMTA, F-54000 Nancy, France
}

\begin{abstract}
Focusing on convective heat transfer modelling upward flame spread scenarios, different methods for calculating the convective heat fluxes are explored. These approaches involve: 1) calculating convective heat transfer based on the stagnant film theory; 2) using a simplified approach based on the stagnant film theory; 3) using Nusselt correlations for natural convection; and 4) modelling convection based on the effective (i.e., laminar plus sgs) thermal diffusivity. Large eddy simulations (LES) of a series of Single Burning Item (SBI) experiments with Medium Density Fiberboard (MDF) and plywood walls are performed. Even though discrepancies are observed, the approach which considered the sgs effects performs overall better and is able to predict both the initial flame growth period and the decay phase with reasonable accuracy (i.e., deviations in the peak HRR of approximately $5 \%$ and $34 \%$ for the MDF and plywood tests cases, respectively). The predicted total heat fluxes are, in most cases, in fairly good agreement with the experiments (i.e., deviations of up to $15 \%$ and $20 \%$ for the MDF and plywood tests cases, respectively). The predictions with the other methods are far less satisfactory. Overall, it is demonstrated that accurate convective heat transfer modelling can be important in flame spread scenarios.
\end{abstract}

Keywords: flame spread, convection, SBI, MDF, plywood, CFD

\footnotetext{
*Corresponding author: Georgios.Maragkos@UGent.be
} 


\section{Introduction}

Boundary layer combustion [1] corresponds to one of the canonical configurations used in fire research and is representative for a variety of fire scenarios. A fundamental understanding of the different physics involved in these scenarios is crucial and many modelling challenges need to be addressed in the context of developing accurate models that could be used towards (predictive) fire modelling. Numerical modelling of boundary layer combustion, within the context of fire applications, remains very challenging due to the intricate coupling of the different modelling aspects (i.e., turbulence, combustion, radiation, soot) involved in the gas phase which cannot be easily decoupled and which strongly affect heat transfer to the solid fuel surface. Even though some challenges still remain (e.g., related to soot modelling), gas phase fire modelling has nowadays reached a certain level of maturity which enables fire research to focus on more intricate fire modelling aspects such as boundary layer combustion. Within this context, accurate modelling of convective and radiative heat transfer is an essential aspect to provide a solid foundation for further modelling of more complex fire scenarios (e.g., involving pyrolysis and flame spread). The fuel gasification rate for pyrolysis is determined by a heat feedback mechanism that depends on convective and radiative heat transfer [2], accurate prediction of which is quite challenging. Firstly, high grid resolutions are typically required in order to resolve the small-scale flow features occurring in the boundary layers, making numerical simulations computationally expensive [3]. Secondly, fires typically involve low-to-moderate Reynolds number flows to which the classical theory of turbulent boundary layers does not apply. Finally, there are multi-physics phenomena involved, e.g, mass transpiration effects (e.g., blowing effects due to solid pyrolysis), turbulence, combustion and radiation, requiring accurate near-surface modelling.

Convective heat transfer is a complex mode of heat transfer, due to the dependency of the heat transfer coefficient on multiple aspects such as, e.g., the case geometry (i.e., vertical, horizontal or more complex surface), type of scenario (i.e., natural, forced or mixed convection) and type of fluid (i.e, gas, liquid). It is well accepted that no universal (empirical) correlation/approach exists for predicting convective heat transfer over all possible scenarios. Modelling of convective heat transfer in fire scenarios involving flammable surfaces becomes even more complex given the additional physics that need to be considered (i.e., mass transpiration effects) and the type of flows typically 
present (i.e., low to moderate Reynolds numbers). Convective heat transfer has been shown to be significant in such scenarios particularly in the downstream flaming zone and during the early stages of flame spread [4]. Most Computational Fluid Dynamics (CFD) codes, often employed to simulate fire scenarios, have been relying on modelling convective heat transfer by employing empirical Nusselt correlations [5] for either natural or forced convection. The limitations of such approaches can be significant and can potentially lead to missing some of the physics involved. Their main disadvantage is that they have been developed for non-reacting flows, considering either isothermal surfaces or constant surface fluxes while ignoring mass transpiration effects (i.e., blowing effect). Additionally, because there is no well-defined free stream flow to be used as input in these correlations, rather the first grid-value is used and the resulting heat transfer rates can be highly grid dependent (i.e., on the choice of the first grid size) [6]. Finally, uncertainties in the order of $25 \%$ have been reported for the resulting heat transfer coefficient values from these correlations depending on the free-stream properties and the surface roughness alone [5]. A different approach for modelling convective heat transfer is often considered in the CFD code FireFOAM [7]. In this case, the maximum value of the convective heat flux is prescribed a-priori (i.e., estimated based on cases without any mass transfer). The approach also considers mass transpiration effects which tend to reduce the convective heat flux in regions where there is mass transfer. Consideration of mass transpiration effects, while modelling of convective heat transfer, has been included to a certain extent in the literature in the past (e.g., $[8,9,10])$ but is not necessarily standard practice in fire modelling (e.g., it is not considered by default in FDS [11]). Additional modelling aspects can also be important with respect to accurate (convective) heat flux predictions. For example, the convective heat transfer was found to be sensitive to the near-wall performance of the turbulence model, while discrepancies in the total heat flux at the bottom of the wall, a region where the flow was quasi laminar and outside the validation range of most sub-grid turbulence models, have also been reported [12]. From the above it is obvious that there are significant uncertainties from the application of some of the aforementioned approaches to fire scenarios involving flammable solid walls. Some of the limitations mentioned are in fact difficult to be circumvented, nevertheless significant improvements are needed in order to enhance convective heat transfer modelling in the context of fire applications.

From employing a boundary layer analysis, for either momentum-driven 
or natural convection flows, it is well established that grid resolutions in the order of millimeters are needed in order to have accurate predictions of convective heat transfer [13]. The required CFD grid resolutions for radiative heat transfer are typically comparable to those needed for accurate fire plume modelling (i.e., in the order of centimeters), hence, are less prohibitive. Nevertheless, grid resolutions in the order of millimeters, even with the advances of today's computer software and technology, are often prohibitive for typical fire scenarios, involving wide range of time and length scales. Within LES, numerical modelling of the boundary layer is typically done either by performing wall-resolved numerical simulations (i.e., well-resolved flow and flame structure near the fuel surface) or through modelling the near-wall region through the use of wall functions and empirical correlations (i.e., unresolved flow and flame structures near the fuel surface). Examples of wall-resolved simulations of turbulent wall fires in the context of LES exist in literature, e.g. $[3,12,14,15]$, reporting on the relative contribution/importance of convective and radiative heat transfer. Numerical studies in which convective heat transfer has been modelled also exist, e.g., $[10,16]$ as well as cases in which flame spread of large-scale configurations has been studied, e.g., $[8,17]$. Yet, it is worth stressing that the relative importance of convective heat transfer has not been excessively studied in literature in the past in the context of flame spread scenarios. Owing to experimental measurement difficulties in obtaining separate heat flux components at the flammable fuel surfaces, there are few experimental data sets available for the evaluation of CFD codes and the available data sets are typically limited to small-scale experiments (e.g., [18]).

The main objective of this article is to investigate the influence of convective heat transfer in scenarios involving flame spread and the development of an accurate, and to the extent possible, grid insensitive approach for modelling convective heat transfer. For this reason, different approaches for modelling convective heat transfer are pursued in order to evaluate their predictive capabilities/accuracy in scenarios involving flame spread: 1) based on the stagnant film theory, 2) a simplified model based on the stagnant film theory, 3) the use of empirical correlations based on the Nusselt number, and 4) based on the effective (i.e., laminar plus sub-grid scale) thermal diffusivity. It is worth noting that modelling of convective heat transfer based on the stagnant film theory has been performed, to some extent, in the past for liquid pool fires and has shown great potential [19]. Nevertheless, the method has not been extensively used for LES of flame spread scenarios, nor 
has it been extensively compared to other methods in literature. This topic is explored in the present work. Correspondingly, a series of Single Burning Item (SBI) experiments with Medium Density Fiberboard (MDF) [20] and plywood [21] walls are considered for evaluation purposes. The present work is a continuation of the authors' efforts on flame spread research and involves well characterised experiments (i.e., in terms of initial and boundary conditions), two types of engineered wood products that are commonly used in the built environment (i.e., making them interesting to study), necessary effective material properties [21] as well as abundance of experimental data that can be used for the evaluation of CFD codes. The work presented in the paper aims to contribute in the context of flame spread modelling, a topic which is of great significance for fire safety engineering and is currently an active area of research in the literature. The activities are a continuation of the work previously performed by the authors on the same topic [9], focusing more on the heat transfer modelling aspect of the problem, aiming to enhance predictive fire modelling when it comes to flame spread.

\section{Models}

The CFD code FireFOAM 2.2.x [7], originally developed by FM Global, is employed here. The code solves the filtered Navier-Stokes equations, along with transport equations for species mass fractions and sensible enthalpy, using Favre-filtered quantities, and employing a unity Lewis number assumption. A detailed presentation of the code and the sub-models has been previously reported (e.g., [22, 23]), hence, only a brief overview of the model choices made is outlined here.

Turbulence is modelled by the dynamic Smagorinsky model [24], calculating the sub-grid scale viscosity as:

$$
\mu_{\text {sgs }}=\bar{\rho}\left(c_{s} \Delta\right)^{2}|\widetilde{S}|
$$

where $\rho$ is the density, $\Delta$ is the filter width (taken as the cube root of the cell volume) and $S$ is the resolved strain rate.

The sub-grid kinetic energy is calculated as:

$$
k_{s g s}=c_{I} \Delta^{2}|\widetilde{S}|^{2}
$$

The sub-grid scale thermal diffusivity is approximated as:

$$
\alpha_{s g s}=\frac{\mu_{s g s}}{P r_{t}}
$$


The sub-grid scale dissipation rate is modelled as:

$$
\epsilon_{s g s}=\frac{c_{\epsilon} k_{s g s}^{3 / 2}}{\Delta}
$$

The model parameters $c_{s}, c_{I}$ and the turbulent Prandtl number, $P r_{t}$, are calculated based on a dynamic procedure [22, 23, 25], and vary locally in time and space, while $c_{\epsilon}=1.0[26]$ is a model constant.

Chemistry is considered to be infinitely fast and turbulence-chemistry interactions are modelled with the Eddy Dissipation Model (EDM) [27] in which the fuel reaction rate is calculated as:

$$
\overline{\dot{\omega}_{F}^{\prime \prime \prime}}=\bar{\rho} \frac{\min \left(\widetilde{Y}_{F}, \widetilde{Y}_{O_{2}} / r\right)}{\tau_{m i x}}
$$

where $Y_{F}$ and $Y_{\mathrm{O}_{2}}$ are the (filtered) fuel and oxygen mass fractions, respectively and $r$ is the stoichiometric oxygen-to-fuel mass ratio.

The mixing time scale is calculated as:

$$
\tau_{m i x}=\frac{k_{s g s}}{A \epsilon_{s g s}}=\frac{1}{A c_{\epsilon} \sqrt{c_{I}} \widetilde{S}}
$$

where $A=2[28]$ is a model parameter which corresponds to an average value across the flame brush.

Radiation is modelled through the finite volume discrete ordinates model (fvDOM). Under the assumption of a non-absorbing, optically thin medium, the radiative heat fluxes are calculated as:

$$
\nabla \cdot \overline{\dot{q}_{r}^{\prime \prime}}=\chi_{r} \overline{\dot{q}_{c}^{\prime \prime \prime}}
$$

where $\chi_{r}$ is a global radiative fraction and $\dot{q}_{c}^{\prime \prime \prime}$ is the local heat release rate per unit volume. The radiative fraction during the numerical simulations is calculated as:

$$
\chi_{r}=\frac{\dot{m}_{\text {burner }} \cdot \chi_{r, \text { burner }}+\dot{m}_{\text {wall }} \cdot \chi_{r, \text { wall }}}{\dot{m}_{\text {burner }}+\dot{m}_{\text {wall }}}
$$

where $\chi_{r}$, burner and $\chi_{r}$, wall are the constant radiative fractions for the propane burner and flammable walls, respectively. Based on the mass flow rate of the propane burner, $\chi_{r}$, burner is approximated as 0.25 [29] while the radiative fractions for the MDF and plywood walls, $\chi_{r}$, wall, are estimated to be in the order of 0.35 (i.e., considering the $\chi_{r}$ values for various wood products 
reported in [30]). No attempt was made to employ more advanced absorption/emission sub-models related to radiation modelling (e.g., WSGGM type of models [31]) given the grid dependency of such approaches on the resolved temperatures and the added need for soot modelling. The decision was made to try and impose the correct amount of heat transferred per unit time by radiation, rather than to perform numerical simulations that are strongly influenced by trying to predict radiative fractions as well.

Focusing on convective heat transfer modelling, different methods are employed for calculating the convective heat fluxes at the flammable walls:

1. Method 1 (named 'SFT' (stagnant film theory)):

$$
\dot{q}_{\text {conv }}^{\prime \prime}=\frac{h}{c_{p}}\left[\left(\frac{\chi_{a}-\chi_{r}}{\chi_{a}}\right) Y_{O_{2}, \infty} \Delta H_{O_{2}}-c_{p}\left(T_{i g n}-T_{\infty}\right)\right] \frac{\dot{m}^{\prime \prime} c_{p} / h}{\exp \left(\dot{m}^{\prime \prime} c_{p} / h\right)-1}
$$

where $\chi_{a}$ is the combustion efficiency of the fuel (taken as 0.79 for MDF and 0.69 for plywood as two softwoods [21]), $\chi_{r}$ is the (global) radiative fraction, $Y_{\mathrm{O}_{2}, \infty}=0.233$ is the ambient oxygen mass fraction, $\Delta H_{\mathrm{O}_{2}}$ is the heat of combustion per $\mathrm{kg}$ of $\mathrm{O}_{2}$ consumed (i.e., $13.1 \mathrm{MJ} / \mathrm{kg}$ ), $c_{p}$ is the heat capacity, $T_{i g n}$ is the ignition temperature of the solid walls (taken as $319^{\circ} \mathrm{C}$ for $\mathrm{MDF}$ and $351^{\circ} \mathrm{C}$ for plywood [21]), $T_{\infty}=20^{\circ} \mathrm{C}$ is the ambient temperature and $\dot{m}^{\prime \prime}$ is the fuel mass loss rate per unit area. This expression essentially stems from the stagnant film theory [32] which describes liquid fuel evaporation due to convective heat transfer from nearby flames. The term $y /(\exp (y)-1)$, with $y=\dot{m}^{\prime \prime} c_{p} / h$, is called the 'blocking factor' and reduces convective heat transfer as mass transfer increases (i.e., termed 'blowing' effect).

The convective heat transfer coefficient, $h$, is calculated based on correlations for laminar and turbulent [33] natural convective burning over vertical surfaces, assuming that $S h=N u$. Here, $h$ is a function of a modified Rayleigh number, $R a_{*}=G r_{*} \cdot \operatorname{Pr}$, calculated as [32]:

$$
\begin{gathered}
N u=\frac{h L}{k}=\frac{0.66 R a_{*}^{1 / 4}}{\Sigma P r^{3 / 4}}, \quad R a_{*} \leq 10^{9} \\
N u=\frac{h L}{k}=\frac{0.0284 R a_{*}^{0.4}}{\sum P r^{2 / 3}}, \quad R a_{*}>10^{9}
\end{gathered}
$$

where

$$
G r_{*}=\left(\frac{L_{v}}{4 c_{p} T_{\infty}}\right)\left(\frac{g L^{3}}{\nu^{2}}\right)
$$




$$
\begin{gathered}
\Sigma=\left[\frac{1+B}{\ln (1+B) B}\right]^{1 / 2}\left[\frac{1+0.5 \operatorname{Pr} /(1+B)}{3\left(B+\tau_{o}\right) \eta+\tau_{o}}\right]^{1 / 4} \\
\tau_{o}=\frac{c_{p}\left(T_{i g n}-T_{\infty}\right)}{L_{v}} \\
\eta=1-\theta_{F O}^{1 / 3} \\
\theta_{F O}=\frac{r_{o}(B+1)}{B\left(r_{o}+1\right)} \\
r_{o}=\frac{Y_{O_{2}, \infty}}{r Y_{F, o}} \\
B=\frac{Y_{O_{2}, \infty} \Delta H_{O_{2}}-c_{p}\left(T_{i g n}-T_{\infty}\right)}{L_{v}}
\end{gathered}
$$

where $L_{v}$ is the latent heat of vaporization of the walls (estimated as $3.2 \mathrm{~kJ} / \mathrm{g}$ for softwood [34]), $\nu$ is the kinematic viscosity, $Y_{F, o}=1$ and $r=1.127$ considering a combustion reaction of pine wood (i.e., $\left.\mathrm{C}_{0.95} \mathrm{H}_{2.4} \mathrm{O}[30]\right)$. The characteristic length, $L$, for natural convection over vertical surfaces is constant and taken as the height of the walls here.

Method 1 'SFT' is not directly affected by the flame temperature, rather only indirectly through the temperature dependency in the estimation of the thermophysical properties (e.g., heat capacity, $c_{p}$, thermal conductivity $k$, kinematic viscosity, $\nu$, and Prandtl number, $\operatorname{Pr}$ ). Hence, the vertical variation of the convective heat fluxes will be due to the variation of the thermophysical properties as well as due to the 'blowing effect' when burning occurs. When there is no (substantial) burning (e.g., during pre-heating of the virgin material and/or early stages of ignition), a fraction of the prescribed value of the maximum convective heat flux is assigned to each computational cell, through a simple linear function, depending on the intensity of the fire in this region (i.e., through examining the resolved convective heat flux) [8, 9]. A detailed description of this approach is described below in method 2 'SSFT'.

2. Method 2 (named 'SSFT'):

- If $\dot{m}_{\text {pyrol }}^{\prime \prime}<10^{-4} \mathrm{~kg} /\left(\mathrm{m}^{2} \cdot \mathrm{s}\right)$ : 


$$
\dot{q}_{\text {conv }}^{\prime \prime}=\frac{\min \left(\max \left(0, \dot{q}_{\text {conv }, \text { laminar }}^{\prime \prime}\right), \dot{q}_{c o n v, \text { threshold }}^{\prime \prime}\right)}{\dot{q}_{\text {conv }, \text { threshold }}^{\prime \prime}} \dot{q}_{c o n v, \text { flame }}^{\prime \prime}
$$

- If $\dot{m}_{\text {pyrol }}^{\prime \prime} \geq 10^{-4} \mathrm{~kg} /\left(\mathrm{m}^{2} \cdot \mathrm{s}\right)$ :

$$
\dot{q}_{\text {conv }}^{\prime \prime}=\dot{q}_{\text {conv }, \text { flame }}^{\prime \prime} \frac{\dot{m}^{\prime \prime} / h_{m}}{\exp \left(\dot{m}^{\prime \prime} / h_{m}\right)-1}
$$

where $\dot{m}_{\text {pyrol }}^{\prime \prime}$ is the mass loss rate from the pyrolyzing wall, $\dot{q}_{c o n v}^{\prime \prime}$ is the computed convective heat flux, $\dot{q}_{\text {conv, laminar }}^{\prime \prime}=-\alpha \rho c_{p} \frac{\partial T}{\partial s}$ is the convective heat flux based on the molecular thermal diffusivity (which is positive when heating of the wall, i.e., $\left.T_{\text {gas }}>T_{\text {walls }}\right), s$ denotes the coordinate normal to the surface of the pyrolyzing surface, $\dot{q}_{\text {conv }}^{\prime \prime}$, threshold an a-priori defined threshold value (i.e., in the range of $0.5-1 \mathrm{~kW} / \mathrm{m}^{2}$ ), $\dot{q}_{\text {conv, flame }}^{\prime \prime}$ is an a-priori prescribed maximum value for the convective heat flux without mass transfer (here set to $15 \mathrm{~kW} / \mathrm{m}^{2}$ assuming an average temperature difference of $1000 \mathrm{~K}$ and a convection coefficient of $\left.15 \mathrm{~W} /\left(\mathrm{m}^{2} \mathrm{~K}\right)\right)$ and $h_{m}$ is a constant mass transfer coefficient approximated as $0.01 \mathrm{~kg} /\left(\mathrm{m}^{2} \mathrm{~s}\right)$ [35]. This approach (which can be viewed as a simplified approach based on a stagnant film theory), often used for modelling convective heat transfer in flame spread scenarios with FireFOAM [7], effectively does not account for the local properties of the flow since it does not involve calculation of the convective heat transfer coefficient, $h$, but assumes the convective heat fluxes to remain relatively constant with elevation. Similarly to method 1, mass transpiration effects are considered (i.e., the final term in the right hand side is the 'blocking factor'). However, contrary to method 1, $h_{m}$ is kept constant. The first part of the wall function is used when there is no (substantial) burning (e.g., during pre-heating of the virgin material and/or early stages of ignition). In this case, a fraction of the prescribed value of the maximum convective heat flux is assigned to each computational cell, through a simple linear function, depending on the intensity of the fire in this region (i.e., through examining the resolved convective heat flux) [8, 9].

3. Method 3 (named 'Nusselt'):

$$
\dot{q}_{\text {conv }}^{\prime \prime}=h\left(T-T_{w}\right)
$$


where the convection coefficient, $h$, is calculated from correlations based on the Nusselt number for laminar and turbulent natural convection at vertical plates [36]:

$$
\begin{array}{ll}
N u=\frac{h L}{k}=0.59 R a^{1 / 4}, & 10^{4} \leq R a, \leq 10^{9} \\
N u=\frac{h L}{k}=0.13 R a^{1 / 3}, & 10^{9}<R a \leq 10^{12}
\end{array}
$$

where $k$ is the temperature-dependent thermal conductivity and $L$ is the characteristic length of the problem (i.e., constant value corresponding to the height of the walls for natural convection problems). The Rayleigh number is defined as $R a=G r \cdot P r$ with the Grashof number calculated as:

$$
G r=\frac{g \beta\left(T-T_{w}\right) L^{3}}{\nu^{2}}
$$

where $g \approx 9.81 \mathrm{~m} / \mathrm{s}^{2}$ is the gravitational acceleration, $\beta=1 / T$ is the volumetric expansion coefficient, $T$ is the gas temperature based on the first grid cell next to the wall, $T_{w}$ is the wall temperature and $\nu$ is the temperature-dependent kinematic viscosity. The first grid cell values next to the wall are used for evaluation of the non-dimensional numbers (i.e., Gr, Pr) and of the convection coefficient. The current approach, often used for modelling convective heat transfer in scenarios not involving flame spread, does not account for any mass transpiration effects which are present when flammable walls are considered. Similar methods are often used for modelling convective heat transfer in fire modelling (e.g., [11]), hence, it is worth investigating how accurate this type of approaches can be in scenarios involving flame spread over combustible walls.

4. Method 4 (named 'Sgs'):

$$
\dot{q}_{c o n v}^{\prime \prime}=\alpha_{e f f} c_{p} \frac{d T}{d n}
$$

where $\alpha_{e f f}=\alpha+\alpha_{s g s}$ is the effective thermal diffusivity, $n$ denotes the direction normal to the wall and $d T / d n$ is the temperature gradient based on the first grid cell temperature and the wall temperature. The molecular thermal diffusivity, $\alpha$, is temperature-dependent while the sub-grid scale thermal diffusivity, $\alpha_{s g s}$, is calculated from the turbulence 
model. The accuracy of this approach will depend not only on the grid size employed but also on the accuracy of the turbulence model in the near-wall region. More specifically, the turbulence model should exhibit decreasing turbulence close to the wall and should be able to predict the transition from laminar to turbulent flow often occurring at the very base of the wall on fire. Neither of these aspects are expected to be accurately captured with the use of static turbulence models. In the limit of DNS, the sub-grid scale contribution in this method would tend to zero corresponding to pure heat transfer by conduction (i.e., Fourier's law). This is the case with the dynamic Smagorinsky model in which the dynamically determined coefficient tends to zero as the filter width approaches the Kolmogorov length scale in contrast with the constant coefficient Smagorinsky model which introduces a fixed error [37].

It is worth noting that verification of the convective heat transfer models presented in the paper has been performed, to the extent possible. More specifically, methods 3 (i.e., 'Nusselt') and 4 (i.e., 'Sgs'), the only methods which are applicable to non-reacting cases and can be validated without the added complexity of involving a fire, were applied to a scenario involving natural convection in a heat cavity [38]. The predicted average Nusselt numbers as well as temperature and velocity profiles at different heights were in fairly good agreement with the experimental data for two different Rayleigh numbers examined. Furthermore, numerical simulations of the same SBI scenarios presented in the paper but involving inert (i.e., calcium silicate) walls [21] have also been performed in order to evaluate the predictive capabilities of all convective heat transfer models in a case which involves a fire but not the added complexity of modelling solid pyrolysis. The predictions of methods 1 (i.e., 'SFT') and 2 (i.e., 'SSFT') were fairly good, in terms of predicted total heat fluxes, and were comparable with the predictions obtained with methods 3 (i.e., 'Nusselt') and 4 (i.e., 'Sgs'). Nevertheless, the results from both numerical studies, mainly performed as part of a verification study of the convective heat transfer models, have not been included here as it is not the main objective of the paper. Studies reporting on verification/validation of the OpenFOAM toolbox / FireFOAM code in different scenarios, involving heat transfer, can be found in literature, e.g., [39, 40, 41, 42].

The thermal decomposition of the solid material is modelled with a simplified one-dimensional pyrolysis model, as described in [43, 44], solving con- 
servation equations for mass and energy using a fully implicit scheme. A single-step Arrhenius pyrolysis reaction is employed considering three species: virgin material, char and pyrolysate. The conservation equations of the pyrolysis model are summarised below.

- Solid reaction

$$
\text { Virgin }=\frac{\rho_{c}}{\rho_{v}} \text { Char }+\left(1-\frac{\rho_{c}}{\rho_{v}}\right) \text { Pyrolysate }
$$

- Energy conservation

$$
\frac{\partial}{\partial t}\left(\rho c_{p} T\right)=\frac{\partial}{\partial x}\left(k \frac{\partial T}{\partial x}\right)+\dot{\omega}_{v}^{\prime \prime \prime} \Delta H_{p}
$$

- Mass conservation

$$
\frac{\partial}{\partial t}\left(\rho Y_{v}\right)=-\dot{\omega}_{v}^{\prime \prime \prime}
$$

- Chemical reaction rate

$$
\dot{\omega}_{v}^{\prime \prime \prime}=\left[\frac{\rho Y_{v}}{\left(\rho Y_{v}\right)_{0}}\right]^{n}\left(\rho Y_{v}\right)_{0} A \exp \left(\frac{E_{a}}{R T}\right)
$$

- Mass loss rate

$$
\dot{m}_{\text {pyrolysate }}=\left(1-\frac{\rho_{c}}{\rho_{v}}\right) \int_{0}^{L} \dot{\omega}_{v}^{\prime \prime \prime} d x
$$

where $\rho_{v}$ and $\rho_{c}$ are the densities of virgin and char, respectively, $Y_{v}$ is the mass fraction of virgin material (subsequently the char mass fraction is $\left.1-Y_{v}\right), \Delta H_{p}$ is the heat of pyrolysis, $n$ is the reaction order, $A$ is the pre-exponential factor, $R$ is the universal gas constant, $E_{a}$ is the activation energy, $T$ is the temperature, $L$ is the solid thickness, $t$ and $x$ are temporal and spatial variables, respectively, while the subscript 0 designates the initial conditions before heating and pyrolysis. The density, $\rho$, and the thermal conductivity, $k$, are bulk values averaged by the volume fractions of virgin and char, while the specific heat, $c_{p}$, is averaged based on mass fractions. The pyrolysis model output is then coupled to the gas phase solver through the specified boundary conditions for temperature, species mass fraction, and velocity. All properties are assumed to be temperature independent while the 
pyrolysate escapes the solid material surface upon formation without any exchange with the solid. The approach employed here also does not consider moisture evaporation or char oxidation. However, their relative influence is indirectly incorporated in the estimation of model-effective material properties presented in Table 1.

In terms of modelling, it is assumed that the released gases from the flammable walls are the same as the fuel of the gaseous burner used in the experiments (i.e., $\mathrm{C}_{3} \mathrm{H}_{8}$ ). Hence, the mass loss rate from the flammable walls, $\dot{m}_{\text {wall }}$, is converted to the equivalent propane fuel in the gas phase as:

$$
\dot{m}_{\text {wall }}=\dot{m}_{\text {pyrolysate }} \frac{\Delta H_{\text {pyrolysate }}}{\Delta H_{\text {propane }}}
$$

where $\dot{m}_{\text {pyrolysate }}$ is the mass flow rate of the pyrolysate (obtained from the pyrolysis model), $\Delta H_{\text {pyrolysate }}$ is the heat of combustion of the pyrolysate (see Table 1) while the heat of combustion of propane is taken as $\Delta H_{\text {propane }}=$ $46.45 \mathrm{MJ} / \mathrm{kg}$.

In order to have a direct comparison between the simulations and the experiments, the total heat fluxes in the simulations are calculated by estimating the total gauge heat fluxes as formulated in [21]:

$$
\dot{q}_{\text {gauge }}^{\prime \prime}=\dot{q}_{\text {inc }}^{\prime \prime}-\sigma T_{\text {gauge }}^{4}+\dot{q}_{\text {conv }}^{\prime \prime}+h\left(T_{w}-T_{\text {gauge }}\right)
$$

where $\dot{q}_{i n c}^{\prime \prime}$ is the incident radiative heat flux, $\dot{q}_{c o n v}^{\prime \prime}$ is the convective heat flux, $\sigma=5.67 \times 10^{-8} \mathrm{~W} / \mathrm{m}^{2} / \mathrm{K}^{4}$ is the Stefan-Boltzmann constant, $h$ is the convection coefficient and $T_{\text {gauge }}=323 \mathrm{~K}$.

\section{Experimental setup}

The Single Burning Item (SBI) experiments [20] considered here, consist of two flammable walls (i.e., a long and a short one) positioned perpendicular to each other forming a corner (see Figure 1). The widths of the long and short walls are $1 \mathrm{~m}$ and $0.5 \mathrm{~m}$, respectively, while their height is $1.5 \mathrm{~m}$. Two different materials are considered, MDF and plywood, with thicknesses of $0.0184 \mathrm{~m}$ and $0.017 \mathrm{~m}$, respectively. The base material of the MDF walls is softwood, containing no phenol resins, manufactured through pressing of fine fibers. The resulting outer surface is a hard, smooth and flat surface while the inner core consists of soft fibers with lower density. The plywood walls consist of 9 plies of softwood species (i.e., conifers) with the face ply made 
Table 1: Model-effective material properties for MDF and plywood used in the simulations.

\begin{tabular}{|c|c|c|}
\hline & $\mathrm{MDF}$ & Plywood \\
\hline \multicolumn{3}{|l|}{ Virgin } \\
\hline Density $\left(\mathrm{kg} / \mathrm{m}^{3}\right)^{a}$ & 605 & 560 \\
\hline Conductivity $(\mathrm{W} / \mathrm{m} / \mathrm{K})^{a}$ & 0.18 & 0.12 \\
\hline Heat capacity $(\mathrm{J} / \mathrm{kg} / \mathrm{K})^{a}$ & 1580 & 1330 \\
\hline Emissivity $^{b}$ & 0.7 & 0.7 \\
\hline \multicolumn{3}{|l|}{ Char } \\
\hline Density $\left(\mathrm{kg} / \mathrm{m}^{3}\right)^{a}$ & 125 & 82 \\
\hline Conductivity $(\mathrm{W} / \mathrm{m} / \mathrm{K})^{a}$ & 0.21 & 0.19 \\
\hline Heat capacity $(\mathrm{J} / \mathrm{kg} / \mathrm{K})^{c}$ & 1450 & 1450 \\
\hline Emissivity $^{d}$ & 0.86 & 0.85 \\
\hline \multicolumn{3}{|l|}{ Reaction } \\
\hline Heat of pyrolysis $(\mathrm{J} / \mathrm{kg})^{a}$ & $1.05 \times 10^{5}$ & $3.5 \times 10^{5 h}$ \\
\hline Act. Energy $(\mathrm{J} / \mathrm{mol})^{a}$ & $7.19 \times 10^{4}$ & $8.01 \times 10^{4}$ \\
\hline Pre-exponential $(1 / \mathrm{s})^{a}$ & $1.47 \times 10^{4}$ & $6.3 \times 10^{4}$ \\
\hline Reaction Order $^{a}$ & 0.66 & 2.94 \\
\hline \multicolumn{3}{|l|}{ Heat of combustion } \\
\hline $\operatorname{Virgin}(\mathrm{J} / \mathrm{kg})^{e}$ & $14.35 \times 10^{6}$ & $13.48 \times 10^{6}$ \\
\hline Char $(\mathrm{J} / \mathrm{kg})^{f}$ & $32.8 \times 10^{6}$ & $32.8 \times 10^{6}$ \\
\hline Pyrolysate $(\mathrm{J} / \mathrm{kg})^{g}$ & $9.54 \times 10^{6}$ & $10.17 \times 10^{6}$ \\
\hline
\end{tabular}

${ }^{a}$ Model effective property estimated based on inverse modelling of FPA experiments in nitrogen [45].

${ }^{b}$ The temperature-dependent emissivity was reported in [45] but an average value is used here based on the spectral range of infrared heaters of FPA.

${ }^{c}$ Specific heat capacity of graphite (carbon) at average surface temperatures of a pyrolyzing sample [43].

${ }^{d}$ Relatively constant for 300 - $3000 \mathrm{~K}$, based on spectral measurements using spectrophotometers [45].

${ }^{e}$ Calculated from the heats of combustion of pyrolysate and char as $\rho_{\text {virgin }} \Delta H_{\text {virgin }}=\left(\rho_{\text {virgin }}-\rho_{\text {char }}\right) \Delta H_{\text {pyrolysate }}+\rho_{\text {char }} \Delta H_{\text {char }}$.

${ }^{f}$ Heat of combustion of carbon.

${ }^{g}$ Based on FPA tests conducted at $50 \mathrm{~kW} / \mathrm{m}^{2}$ in normal atmosphere.

${ }^{h}$ The inverse modelling results suggested a value of $-2.15 \times 10^{5} \mathrm{~J} / \mathrm{kg}$, indicating an exothermic reaction in the nitrogen atmosphere. For the normal atmosphere of the fire experiments, it was deemed more appropriate to consider an endothermic value, estimated as $\Delta H_{p}=L_{v}-c_{p}\left(T_{i g n}-T_{\infty}\right)$. 


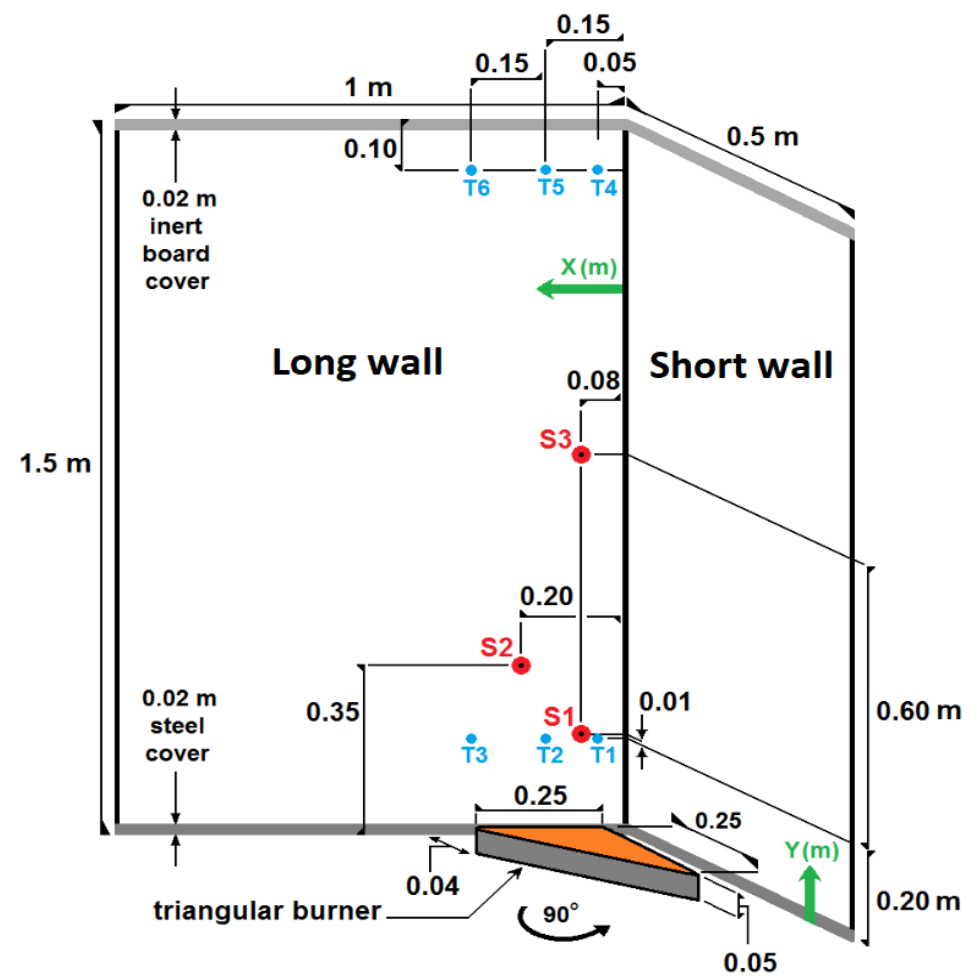

Figure 1: Illustration of the setup used in the SBI experiments [20, 21]. The locations of the measured total heat fluxes and the backside wall temperatures are denoted as S1-S3 and T1-T6, respectively. Figure reproduced from [9].

of cedar. Bonding of the plies has been achieved with a water boiled proof phenol formaldehyde glue making the walls highly resistant to delamination [21]. The corner fire source is a $30 \mathrm{~kW}$ triangular propane $\left(\mathrm{C}_{3} \mathrm{H}_{8}\right)$ burner with side dimension of $0.25 \mathrm{~m}$, located at a clearance of $0.04 \mathrm{~m}$ from the walls, with a $30 \mathrm{~s}$ ramp-up time [9]. The measurements considered include the heat release rate (HRR), obtained from oxygen calorimetry, the total heat fluxes at 3 locations on the long walls, obtained using water-cooled Schmidt-Boelter heat flux sensors, and the backside temperatures at 6 different locations on the long walls, obtained using K-type thermocouples (0.0005 m diameter). The temperature of the cooling water supplied to the sensors was maintained at $50{ }^{\circ} \mathrm{C}$ (i.e., $323 \mathrm{~K}$ ) to diminish condensation errors. The exact locations of the measured heat fluxes and the backside temperatures are shown in Figure 1. More information regarding the setup and the measuring techniques 
employed in the experiments can be found in [20, 21].

\section{Numerical setup}

A rectangular computational domain of $1 \mathrm{~m} \times 2 \mathrm{~m} \times 1 \mathrm{~m}$ (length $\mathrm{x}$ height $\mathrm{x}$ width) is used to model the case. A local mesh refinement strategy is employed with grid sizes ranging between $1 \mathrm{~cm}$ to $4 \mathrm{~cm}$ depending on the location inside the computational domain (Figure 2). A grid size of $1 \mathrm{~cm}$ is employed in the main regions of interest, i.e., in the corner and adjacent to the flammable walls all the way up to their height. The refinement has been used up to $0.5 \mathrm{~m}$ from the corner (i.e., fully covering the short wall and half covering the long wall) because that is where most of the burning occurs. Outside the burner area the mesh is set to $2 \mathrm{~cm}$ and towards the opposite corner of the walls (i.e., open boundaries), a grid size of $4 \mathrm{~cm}$ is used. The gas phase cells are mapped onto the solid walls with 45 cells considered through the thickness of the walls. The total number of cells in the gas and solid phase is approximately 280000 and 750000 , respectively. The chosen grid size is sufficiently small for accurate fire plume modelling (i.e., in the order of centimeters) but not small enough for resolving the boundary layer (i.e., in the order of millimeters [3]). This choice was made intentionally in order to evaluate the different methods for convective heat transfer modelling on grid sizes that are typical for modelling fire scenarios.

The corresponding mass flow rate (accounting for both the convective and diffusive mass fluxes) is applied at the propane burner with a prescribed time ramp to reach the maximum HRR (i.e., $30 \mathrm{~kW}$ ) after approximately $30 \mathrm{~s}$, according to the experiments. The ambient temperature and pressure are set to $293 \mathrm{~K}$ and $101325 \mathrm{~Pa}$, respectively. A PIMPLE algorithm is used for the pressure-velocity coupling with a Rhie-Chow interpolation to avoid odd-even decoupling. A mixed boundary condition is assigned for velocity at the open boundaries of the computational domain, setting zero gradient for any outward flow and calculating the inlet velocity from pressure. A Dirichlet boundary condition is assigned for pressure at the open boundaries of the computational domain, which fixes total pressure, and when velocity changes, the dynamic pressure is adjusted accordingly. At the outlet (i.e., top boundary), a Neumann (i.e., zero gradient) boundary condition is assigned to all variables with no reverse flow allowed in order to avoid numerical instabilities. The different approaches for modelling convective heat transfer are implemented through the prescribed boundary condition for the sub-grid 


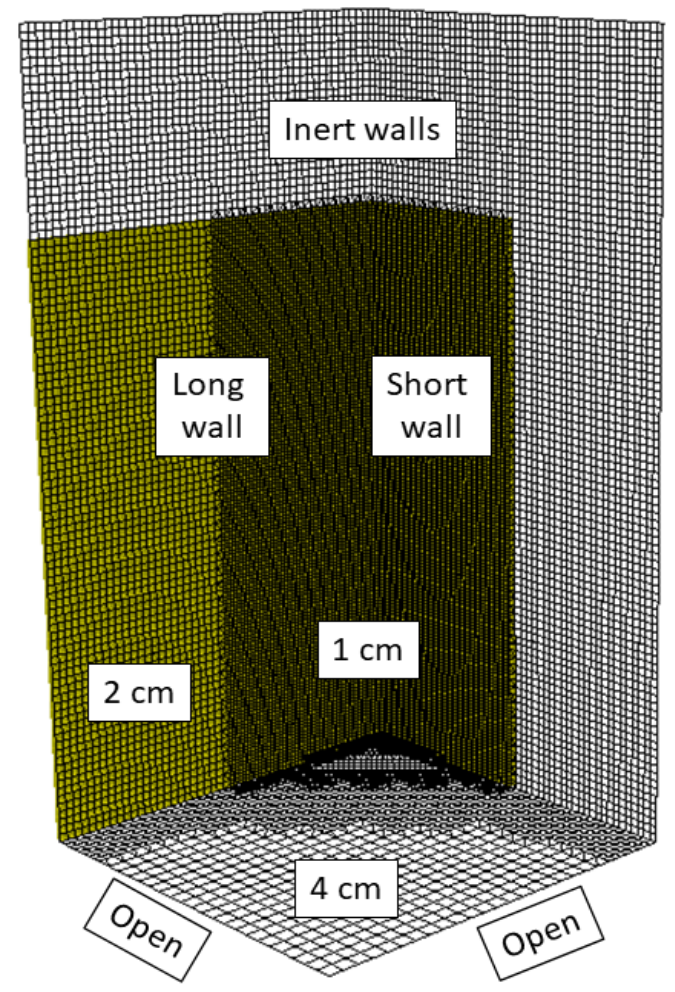

Figure 2: Computational domain used in the numerical simulations showing the different levels of grid refinement and the position of the flammable walls.

scale thermal diffusivity, hence, it changes depending on the method employed. The solid surfaces outside the flammable walls are treated as inert with a no-slip boundary condition applied for velocity, a small value (i.e., $10^{-6} \mathrm{~m}^{2} / \mathrm{s}^{2}$ ) assigned for sub-grid scale kinetic energy and a zero gradient boundary condition for the chemical species. Heat losses from the backside of the walls are considered with a constant convective heat transfer coefficient of $15 \mathrm{~W} /\left(\mathrm{m}^{2} \mathrm{~K}\right)$. In the absence of an available boundary condition in the CFD code, this value was set in order to account for any radiative losses from the backside of the walls as well (which could not have been accounted for otherwise). For the solution of the RTE, 72 solid angles are considered for angular discretization. The equations are advanced in time using a first order Euler scheme. For the convective terms a second order central difference scheme (i.e., Gauss linear) is employed while for scalar transport a TVD scheme (i.e., limitedLinear) is used. For the diffusive terms a central differ- 
ence scheme is applied while for the discretization of the RTE a first order upwind scheme is used. The simulations are run for $900 \mathrm{~s}$ with a variable time step, corresponding to a maximum Courant number of 0.9. A parallel decomposition strategy is employed by dividing the computational domain across 24 processors using a scotch algorithm which optimises the decomposition process in order to have minimal communication among processors. The total physical running time was $135 \mathrm{~h}$ on a Dell PowerEdge R630 server with 2 x 12-core Intel Xeon E5-2680v3 $2.5 \mathrm{GHz}$ with 128GB RAM.

\section{Results}

\subsection{Total heat release rates}

Figure 3 presents the comparison between the experiments and the predicted total HRR from the numerical simulations with the MDF and plywood walls, focusing on the influence of the different methods employed for modelling convective heat transfer. The lines present simulation results while the symbols denote experimental data from the different tests, which also give an indication of the repeatability of the experiments.

Starting with the MDF walls (Figure 3(a)), the observed increase in the total heat release rate in the first $40 \mathrm{~s}$ (i.e., $30 \mathrm{~kW}$ ) is mainly due to the contribution of the propane burner: during this period the virgin material is still heating up without undergoing any significant pyrolysis. After this time the contribution to the heat release rate is due to the pyrolyzing walls, releasing flammable gases and burning occurring mainly in the vicinity of the corner. A first peak in the HRR (i.e., $120 \mathrm{~kW}$ ) is observed in the experiments around $90 \mathrm{~s}$ followed by a decrease until the HRR reaches its maximum value, of approximately $160 \mathrm{~kW}$, at about $155 \mathrm{~s}$. This initial decrease in the HRR was attributed to the burning of the virgin material which led to the formation of a char layer at the surface of the wall which restricted heat transfer through its thickness (i.e., given the lower conductivity of char compared to that of virgin material [20]. Subsequently, there was delamination of the char layer which allowed virgin material to be exposed to heating from the flames and further continuation of the burning of the walls (i.e., hence the increase in the HRR around $100 \mathrm{~s}$ ). It is worth noting that delamination effects are not considered in the pyrolysis model, hence, this aspect cannot be captured by the numerical simulations. After the maximum peak HRR is reached there is a gradual decrease in the burning until the end of the test (i.e., at $900 \mathrm{~s}$ )

with the resulting HRR in the order of $65-70 \mathrm{~kW}$. Towards the end of the 


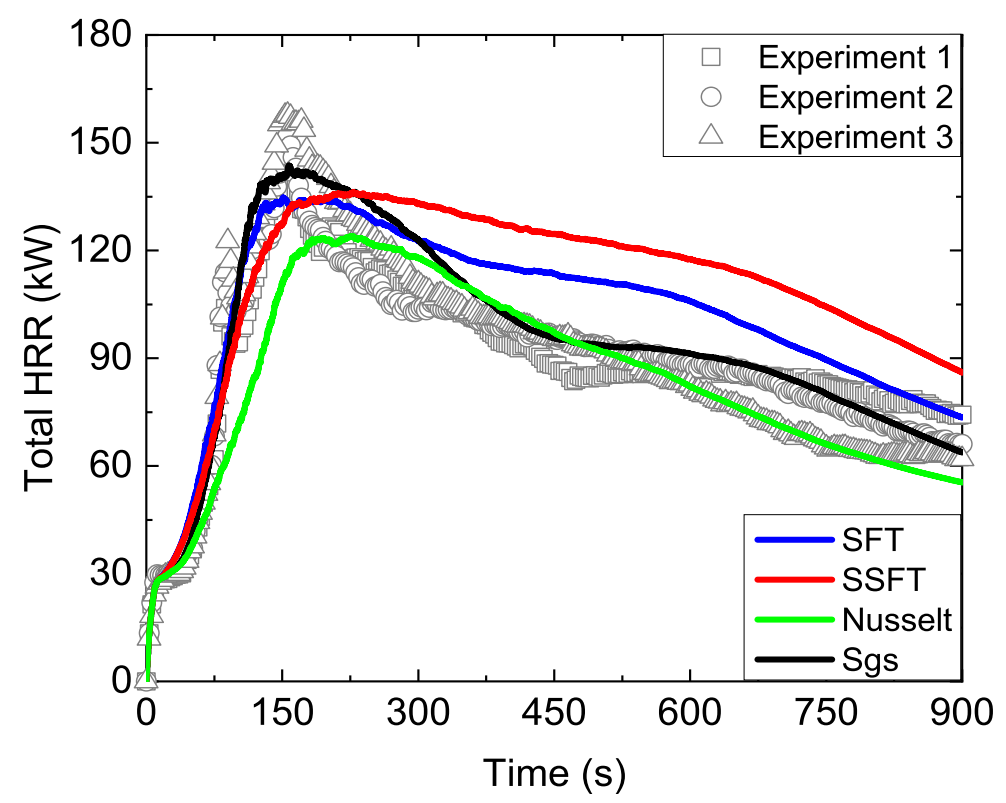

(a)

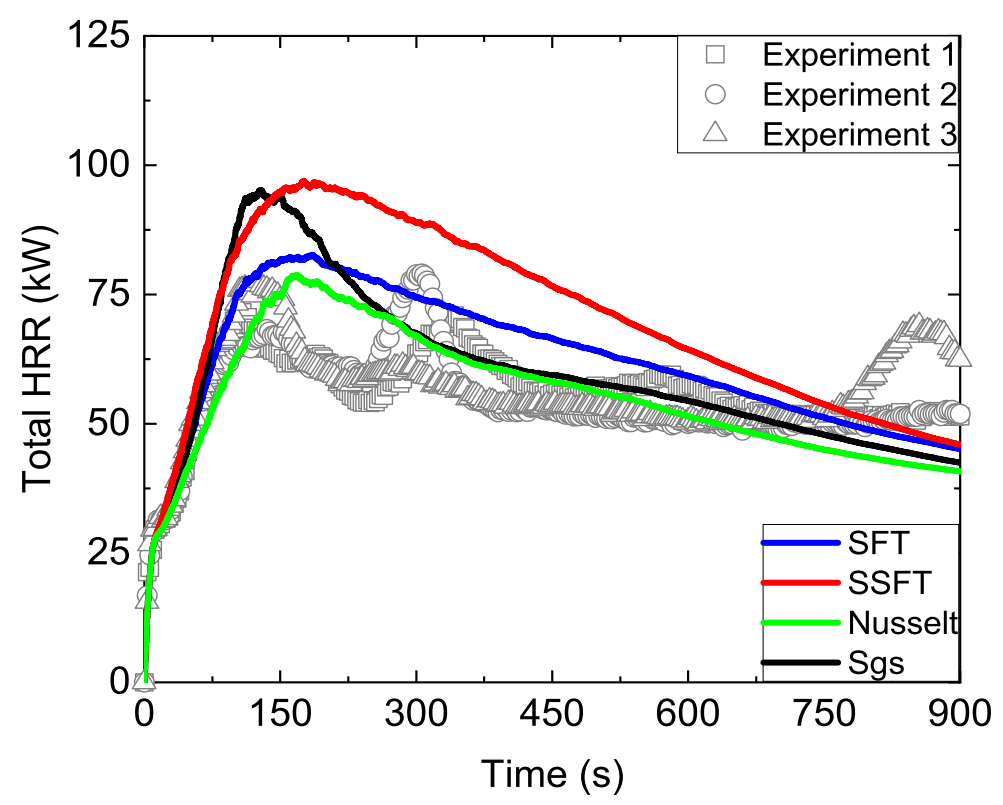

(b)

Figure 3: Temporal evolution of total HRR with the (a) MDF and (b) plywood walls with a grid size of $1 \mathrm{~cm}$. Methods: 1 -'SFT', 2 -'SSFT', 3 -'Nusselt', 4 -'Sgs'. 
experiments, it is expected that some small contribution from char oxidation will be present in the total heat release rate. To avoid added modelling uncertainty, char oxidation was not modelled in the present study. Even though this approach is not perfect, it is considered as first step before attempting to model the complex process of char oxidation in scenarios involving flame spread. Similarly, the modelling of char cracking and delamination in the MDF experiments [21] was considered to be beyond the scope of the present study. An overview of some characteristic quantities predicted in the simulations with the MDF and plywood walls (i.e., the time to peak HRR, the peak HRR and the HRR at a representative time for the decay phase (i.e., at $t=800 \mathrm{~s}$ )) as well as their deviations from the experiments is outlined in Table 2 in order to have a more quantitative measure of the performance of the different methods.

Table 2: Selected characteristic quantities (i.e., $t \rightarrow H \rightarrow R_{\text {max }}$ : time to peak HRR, $\mathrm{HRR}_{\text {max }}$ : peak HRR, $\mathrm{HRR}_{t=800 \mathrm{~s}}$ : HRR at a representative time for the decay phase) predicted in the simulations as well as their deviations from the experiments for the MDF and plywood walls. The experimental data are based on the average of 3 tests.

\begin{tabular}{|c|c|c|c|}
\hline & $\mathrm{t} \rightarrow \mathrm{HRR}_{\max }$ & $\mathrm{HRR}_{\text {max }}$ & $\mathrm{HRR}_{t=800 \mathrm{~s}}$ \\
\hline \multicolumn{4}{|l|}{ Experiments } \\
\hline MDF & $152 \mathrm{~s}$ & $151 \mathrm{~kW}$ & $72 \mathrm{~kW}$ \\
\hline Plywood & $105 \mathrm{~s}$ & $71 \mathrm{~kW}$ & $53 \mathrm{~kW}$ \\
\hline \multicolumn{4}{|l|}{ Method 1 ('SFT') } \\
\hline $\mathrm{MDF}$ & $151 \mathrm{~s}(-0.7 \%)$ & $135 \mathrm{~kW}(-11 \%)$ & $84 \mathrm{~kW}(+17 \%)$ \\
\hline Plywood & $185 \mathrm{~s}(+76 \%)$ & $83 \mathrm{~kW}(+17 \%)$ & $49 \mathrm{~kW}(-7 \%)$ \\
\hline \multicolumn{4}{|l|}{ Method 2 ('SSFT') } \\
\hline $\mathrm{MDF}$ & $228 \mathrm{~s}(+50 \%)$ & $136 \mathrm{~kW}(-10 \%)$ & $98 \mathrm{~kW}(+36 \%)$ \\
\hline Plywood & $176 \mathrm{~s}(+68 \%)$ & $97 \mathrm{~kW}(+37 \%)$ & $51 \mathrm{~kW}(-4 \%)$ \\
\hline \multicolumn{4}{|l|}{ Method 3 ('Nusselt') } \\
\hline $\mathrm{MDF}$ & $225 \mathrm{~s}(+48 \%)$ & $124 \mathrm{~kW}(-18 \%)$ & $62 \mathrm{~kW}(-14 \%)$ \\
\hline Plywood & $163 \mathrm{~s}(+55 \%)$ & $79 \mathrm{~kW}(+11 \%)$ & $43 \mathrm{~kW}(-19 \%)$ \\
\hline \multicolumn{4}{|l|}{ Method 4 ('Sgs') } \\
\hline $\mathrm{MDF}$ & $158 \mathrm{~s}(+4 \%)$ & $144 \mathrm{~kW}(-5 \%)$ & $75 \mathrm{~kW}(+4 \%)$ \\
\hline Plywood & $128 \mathrm{~s}(+22 \%)$ & $95 \mathrm{~kW}(+34 \%)$ & $46 \mathrm{~kW}(-13 \%)$ \\
\hline
\end{tabular}

Overall, the best predictions are obtained with method 4 (i.e., 'Sgs'), 
capturing quite accurately the initial flame growth period and the subsequent decrease in the heat release rate (i.e., decay phase) as a function of time. Small discrepancies are present (i.e., in the order of up to 5\%) but given the simplifications present in the modelling the predictions are considered very satisfactory. Method 1 (i.e., 'SFT') performs less accurately: even though it captures the initial flame growth period fairly well, it underestimates the peak HRR (i.e., by approximately $11 \%$ ) and remains at too high values during the decay phase (starting from $300 \mathrm{~s}$ until $750 \mathrm{~s}$ in the experiments). A possible reason for the deficiencies observed in this case could be the use of a constant value for the B number [46] in Eq. (18) because the heat of vaporization (gasification), $L_{v}$, can vary depending on several aspects among which can be e.g., the grain direction [47] and the developed char depth [48]. The consideration of a variable B number, that would change in time and space, would have an indirect effect on the convective heat fluxes (i.e., increasing B values reduce $\dot{q}_{\text {conv }}^{\prime \prime}$ ) and would eventually affect the heating of the virgin material as well as the resulting burning of the walls. This aspect needs further investigation in the future. Qualitatively, similar observations are made for method 2 (i.e., 'SSFT'). In this case, the use of a constant value for the maximum convective heat flux (i.e., $\dot{q}_{\text {conv, flame }}^{\prime \prime}$, regardless of the spatial location on the walls, is not realistic. Given its formulation, variation of the convective heat fluxes with method 2 will mainly occur due to mass transpiration effects (i.e., when the walls will start burning) without accounting for the local flow properties. In addition, a constant value for the convective heat flux when no mass transfer effects are present (i.e., $\dot{q}_{\text {conv, flame }}^{\prime \prime}$ value) over the whole geometry is not totally realistic. These aspects can result in significant pre-heating of the virgin wall in the early stages of the tests which eventually results in more intense burning in the later stages. The predictions using method 3 (i.e., 'Nusselt') are, overall, better than those of method 1 (i.e., 'SFT'); even though in less good agreement with the experimental data during the initial flame growth period, they perform significantly better in the later decay phase (see selected deviations presented in Table 2). Differences in the convective heat fluxes in the early stages of the simulations, where method 3 (i.e., 'Nusselt') predicted lower convective heat fluxes than method 1 (i.e., 'SFT') (see results later), could explain the differences in the predicted HRR and in the overall burning behaviour between the two methods. Given the similarities in the predictions between method 3 (i.e., 'Nusselt') and method 4 (i.e., 'Sgs'), which both consider a temperature difference for the estimation of the convective heat fluxes, the 
main reason for any inaccuracies (i.e., related to the early stages of flame spread) observed with method 3 (i.e., 'Nusselt'), could be attributed to the possible invalidity of the employed Nusselt correlations in scenarios for which they have not been developed : they have been developed for non-reacting flows without mass transfer, while here we have reacting flows involving mass transfer. In this regard, it is worth noting that method 3 (i.e., 'Nusselt') neglects the 'blowing effect' (i.e., reduction of the convective heat fluxes in the presence of mass transfer) which would decrease the convective heat fluxes, hence, result in even lower HRR than what is now predicted. For this reason, the predictions with method 3 (i.e., 'Nusselt') are in fact better than what they initially appear to be, due to compensating effects: any grid dependency or inaccuracy of the employed Nusselt correlations that could potentially lower the convective heat fluxes is essentially canceled by neglecting the 'blowing effect'.

The cases with the plywood walls (Figure 3(b)) involve less intensive burning (i.e., maximum total HRR of approximately $71 \mathrm{~kW}$ at about 105 s) and also exhibit significantly less surface delamination in the experiments [21] compared to the MDF cases. After the initial flame growth period, the HRR remains relatively constant at approximately $50 \mathrm{~kW}$ until the end of the tests. It is worth noting that the contribution from the burning plywood walls in this case is less than $30 \mathrm{~kW}$ on average (with the propane burner having an output of $30 \mathrm{~kW}$ ) signifying that there is no substantial burning of the plywood walls. The main differences between method 1 (i.e., 'SFT'), method 3 (i.e., 'Nusselt') and method 4 (i.e., 'Sgs') lie in the initial flame growth period (i.e., peak HRR value and time to peak HRR) since later on (i.e., from $300 \mathrm{~s}$ onwards until the end of the simulations) all methods have similar predictions. Overall, method 1 (i.e., 'SFT') predicts the value of the initial peak HRR fairly well (i.e., $+17 \%$ ), however, the time to peak HRR is significantly delayed (i.e., $+76 \%$ ). Method 3 (i.e., 'Nusselt') also predicts the peak HRR reasonably (i.e., $+11 \%$ ) but with some time delay (i.e., $+55 \%$ ), while method 4 (i.e., 'Sgs') predicts the time to peak HRR fairly reasonably (i.e., $+22 \%$ ) but overestimates the peak HRR value (i.e., $+34 \%$ ). The predictions with method 2 (i.e., 'SSFT') are, overall, not satisfactory as not only they overestimate the peak HRR value in the initial flame growth period (i.e., $+37 \%$ ) and the time to reach the peak HRR (i.e., $+68 \%$ ), but also result in more intense burning until almost the end of the simulations. The predictions with the various methods in this case essentially follow the pattern previously observed for the MDF case as well (e.g., overestimated 
HRR after the peak HRR is reached with method 1 (i.e., 'SFT'), more intense burning in general with method 2 (i.e., 'SSFT'), slower increase of the HRR in the initial flame spread period with method 3 (i.e., 'Nusselt') and fairly accurate predictions with method 4 (i.e., 'Sgs') even though the peak HRR in this case is overestimated).

\subsection{Mesh sensitivity}

In order to investigate the effect of grid size in the simulation, selected results including the total heat release rate (i.e., burner plus flammable walls), shown in Figure 4 and Figure 5 for the MDF and plywood cases, respectively, as well as the individual heat flux components at two different locations (i.e., sensors S1 and S2), shown in Figures 6-9 for the MDF case, are presented for three different grid sizes (i.e., $4 \mathrm{~cm}, 2 \mathrm{~cm}$ and $1 \mathrm{~cm}$ ). Reasonably grid independent results were obtained for a finer grid size of $0.5 \mathrm{~cm}$ (i.e., deviations within 10\%). The grid sensitivity on the heat fluxes for the plywood case has been omitted to avoid repetition as the observations were similar to the ones observed for the MDF case. The scatter in the experimental data illustrates the repeatability level of the tests.

By examining the predicted HRR for the MDF and the plywood cases, shown in Figures 4 and 5, respectively, it is evident that the influence of the grid size is substantial on the coarsest grid size (i.e., $4 \mathrm{~cm}$ ) employed in the simulations, which is therefore confirmed to be too coarse here (which is not surprising, as it would even be too coarse for fire plume modelling with this burner). When the coarsest grid size is employed, all approaches fail to capture the initial flame growth period (i.e., for both the MDF and plywood cases) and significantly underestimate the burning of the flammable walls throughout the entire duration of the test (i.e., for the MDF case). The differences between the predictions with the $2 \mathrm{~cm}$ and $1 \mathrm{~cm}$ grid sizes are smaller, and even though still considerable (i.e., in the order of up to $30 \%$ ), in most cases they tend to follow the experimental patterns of the burning walls qualitatively. As expected, the heat release rate using the $1 \mathrm{~cm}$ grid size is higher, compared to the $2 \mathrm{~cm}$ grid size, due to more accurate (convective and radiative) heat transfer predictions and better resolution of the flames in the near-wall region, enhancing the burning of the flammable walls. Even though differences are present (i.e., in the peak HRR, the time to peak HRR and in the decay phase of the HRR), the predicted burning behaviour with methods 1 (i.e., 'SFT') , 3 (i.e., 'Nusselt') and 4 (i.e., 'Sgs') is qualitatively similar, as

opposed to method 2 (i.e., 'SSFT') in which the HRR in the decay phase is 


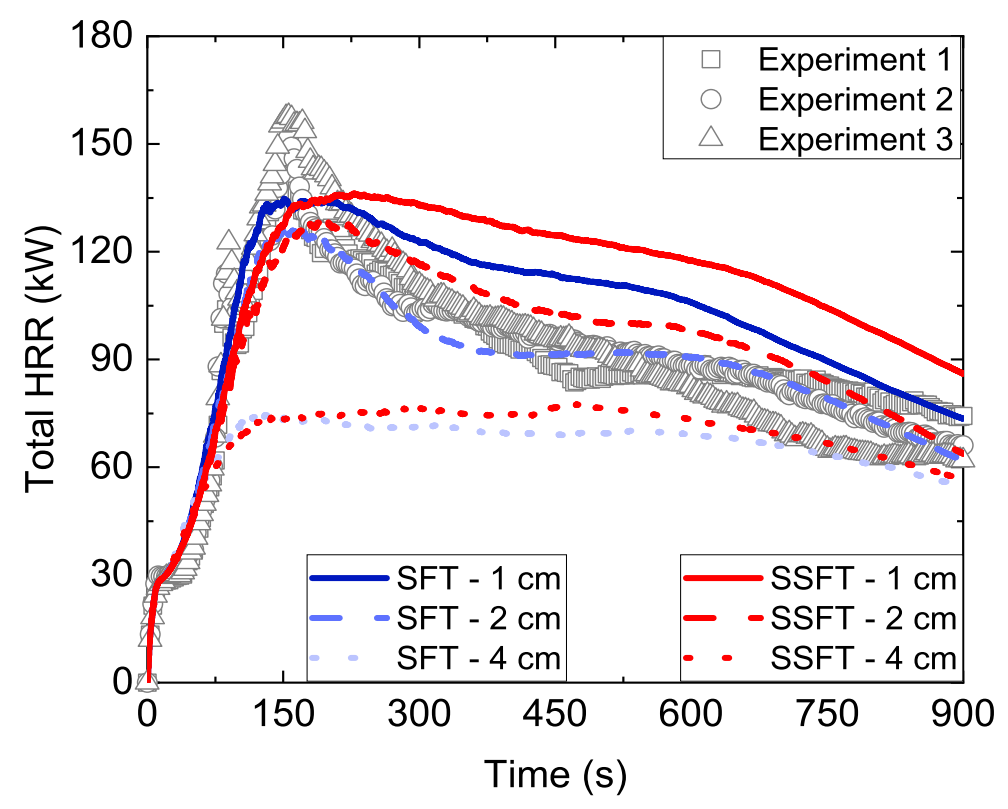

(a)

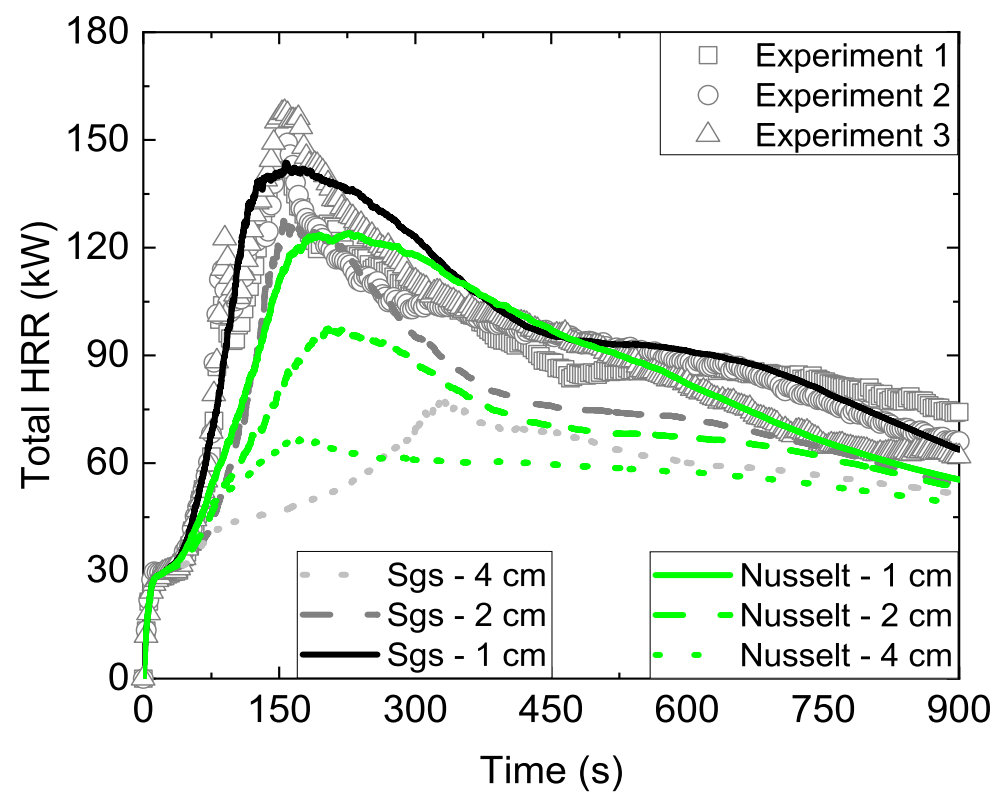

(b)

Figure 4: Temporal evolution of total HRR for the MDF walls with method (a) 1-'SFT' and 2-'SSFT' and (b) 3-'Nusselt' and 4-'Sgs' as a function of grid size. The scatter in the experimental data illustrates the repeatability level of the tests. 


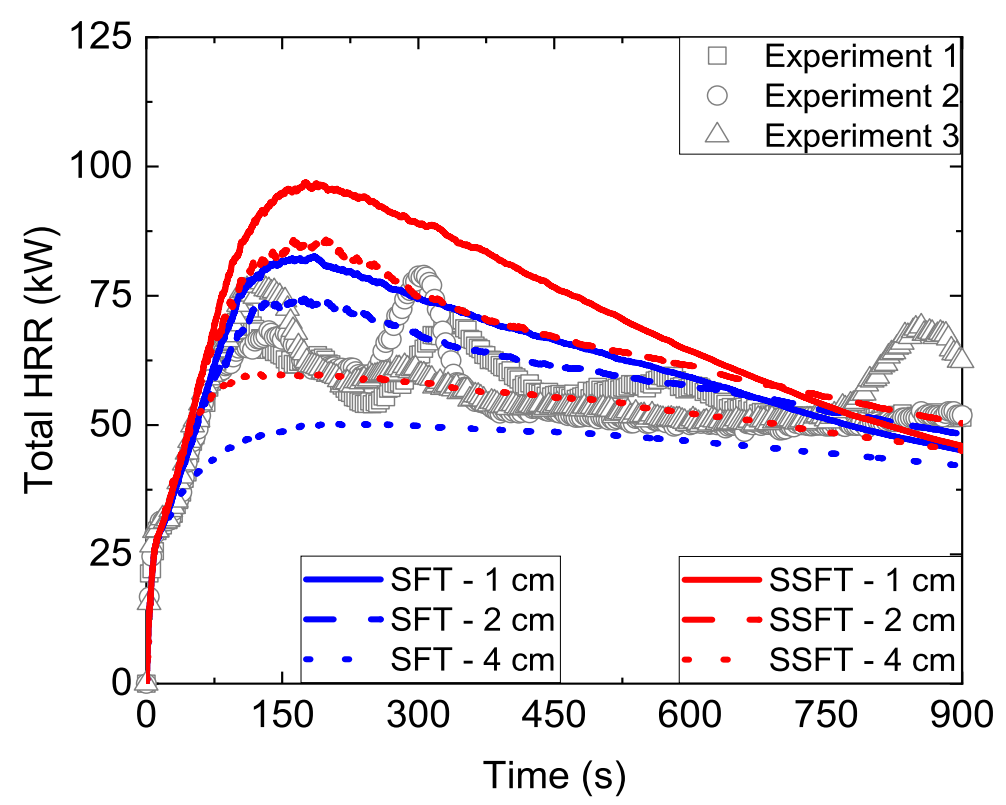

(a)

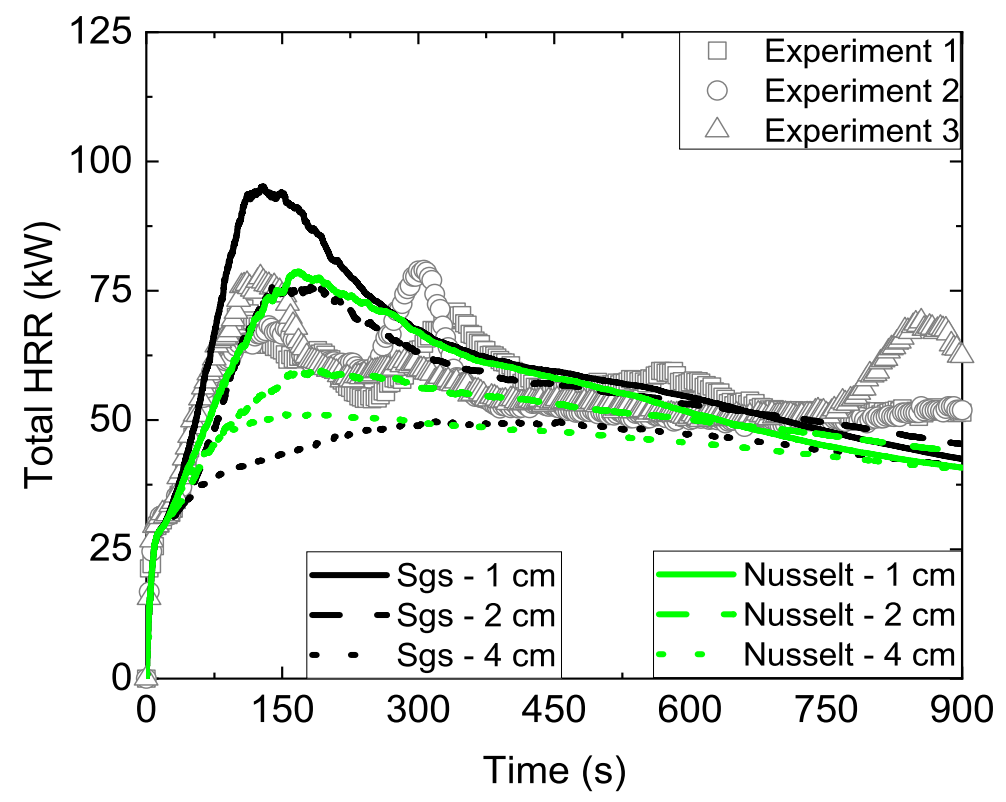

(b)

Figure 5: Temporal evolution of total HRR for the plywood walls with method (a) 1-'SFT' and 2-'SSFT' and (b) 3-'Nusselt' and 4-'Sgs' as a function of grid size. The scatter in the experimental data illustrates the repeatability level of the tests. 
overestimated in both cases. From all the methods tested, methods 3 (i.e., 'Nusselt') and 4 (i.e., 'Sgs') exhibit the strongest grid dependency due to the calculation of a temperature difference in the estimation of the convective heat fluxes. Part of the differences in the burning behaviour between the different methods can be further explained by examining the individual heat flux components, shown below in Figures 6-9.

Overall, the predictions between methods 1 (i.e., 'SFT') and 2 (i.e., 'SSFT') are qualitatively similar when examining the predicted convective heat fluxes at sensors S1 (Figure 6) and S2 (Figure 7). Given their formulation, both methods will typically predict a relatively constant convective heat flux unless significant burning occurs and the effect of mass transfer becomes important (i.e., reduction in the convective heat fluxes will mainly occur due to the 'blowing' effect). Nevertheless, local effects are considered indirectly in method 1 (i.e., 'SFT') through the temperature dependency of pyrolysis. Similarly, the predictions between methods 3 (i.e., 'Nusselt') and 4 (i.e., 'Sgs') are also comparable. In this case, both methods account for the local flow properties in a more realistic way than methods 1 (i.e., 'SFT') and 2 (i.e., 'SSFT') (e.g., by considering the local temperature despite any deficiencies this aspect might have) and result in convective heat fluxes which increase with increasing levels of turbulence. A first important observation is that the convective heat fluxes with methods 1 (i.e., 'SFT') and 2 (i.e., 'SSFT') are significantly less grid sensitive than with methods 3 (i.e., 'Nusselt') and 4 (i.e., 'Sgs'), at sensor S1 (Figure 6) and S2 (Figure 7). This is to be expected as both methods do not explicitly involve the calculation of the temperature difference for the estimation of the convective heat fluxes. In general, all methods predict a quick increase in the convective heat fluxes at sensor S1 (Figure 6), due to convective heating from the flames of the propane burner, followed by a decrease once significant burning starts to occur (i.e., when the peak HRR is reached) and mass transpiration effects become important. After the peak HRR is reached (i.e., at about $150 \mathrm{~s}$ ), methods 1 (i.e., 'SFT'), 3 (i.e., 'Nusselt') and 4 (i.e., 'Sgs') predict constant convective heat fluxes as opposed to method 2 (i.e., 'SSFT') in which the convective heat fluxes start to increase again. This is due to the fact that a constant

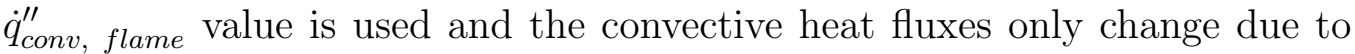
mass transpiration effects, not accounting for local flow properties. Focusing on the results at sensor S2 (Figure 7), the predicted convective heat fluxes do exhibit now a significant grid dependency, particularly with method 3 (i.e., 'Nusselt') and followed by method 4 (i.e., 'Sgs'), where the strongest 


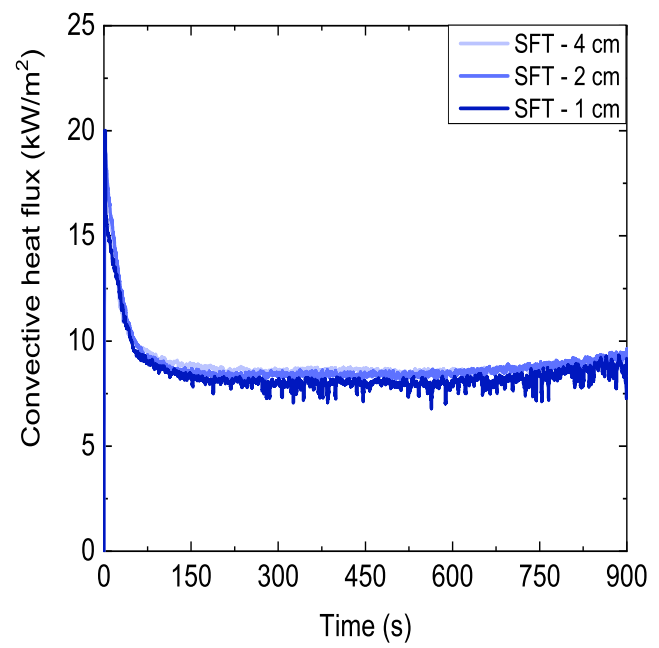

(a)

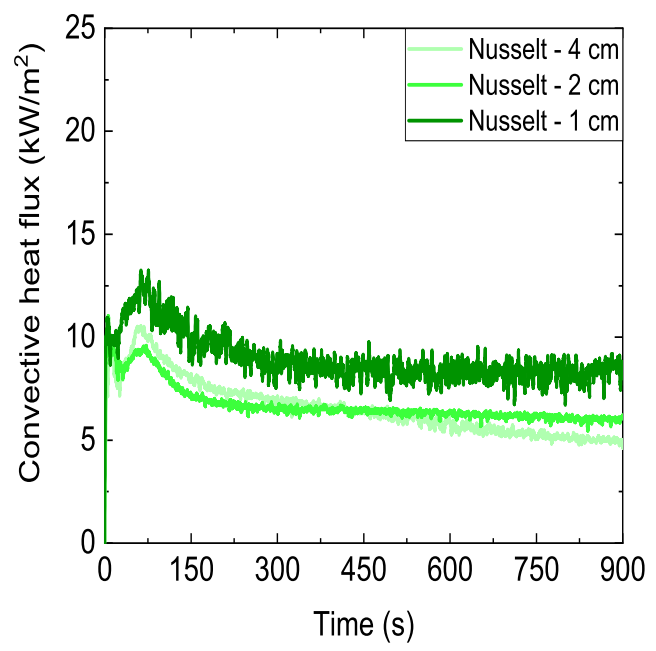

(c)

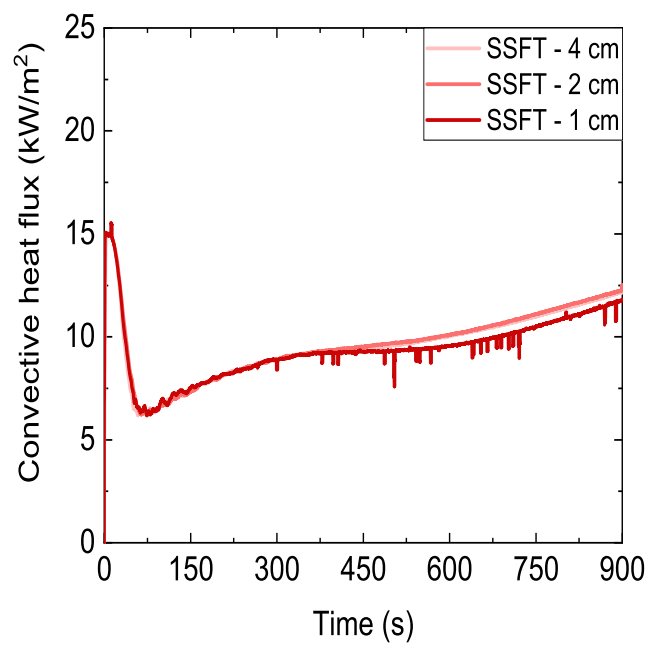

(b)

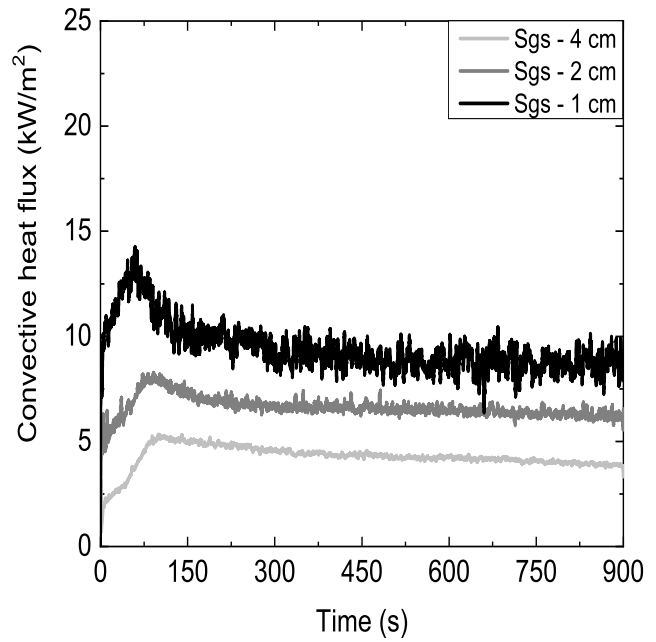

(d)

Figure 6: Temporal evolution of the predicted convective heat fluxes at sensor S1 with method (a) 1-'SFT', (b) 2-'SSFT', (c) 3-'Nusselt' and (d) 4-'Sgs' for the MDF walls as a function of grid size. 


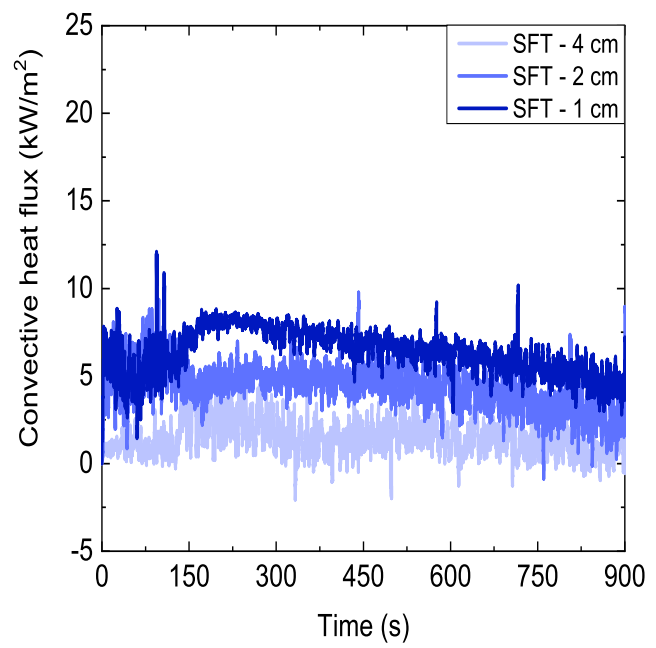

(a)

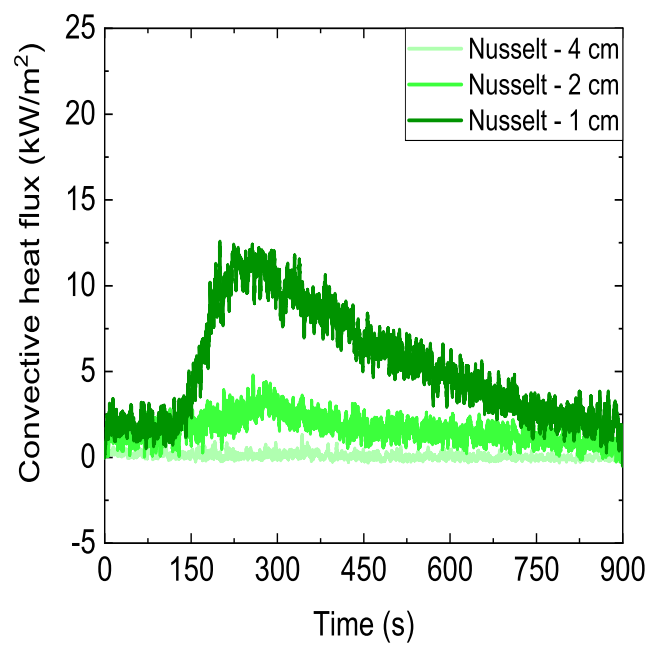

(c)

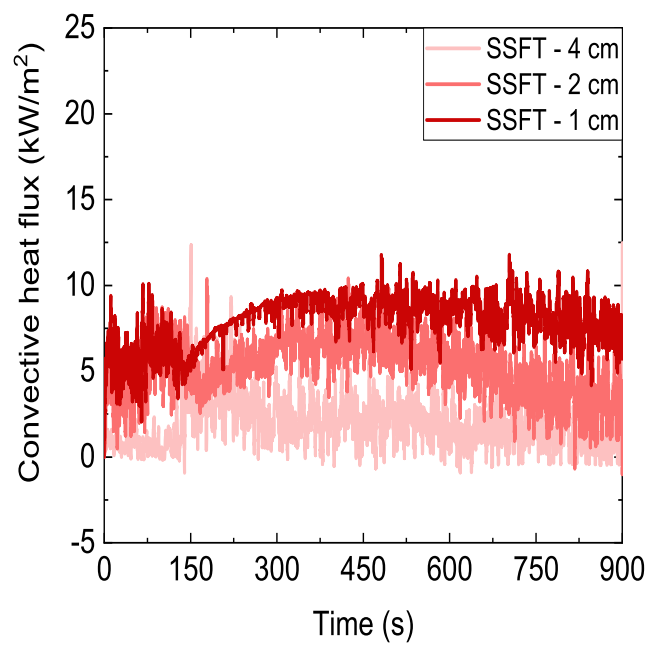

(b)

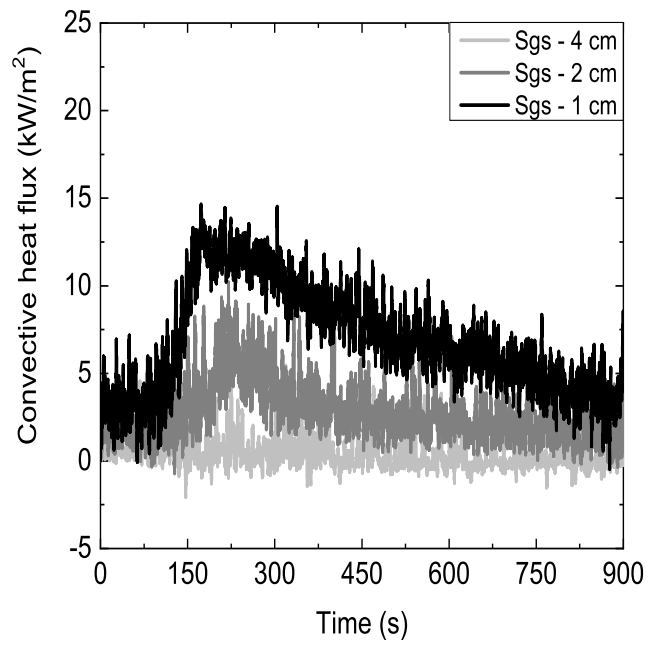

(d)

Figure 7: Temporal evolution of the predicted convective heat fluxes at sensor S2 with method (a) 1-'SFT', (b) 2-'SSFT', (c) 3-'Nusselt' and (d) 4-'Sgs' for the MDF walls as a function of grid size. 
differences are observed between the $1 \mathrm{~cm}$ and $2 \mathrm{~cm}$ mesh results (the $4 \mathrm{~cm}$ mesh results should not be considered, as mentioned before). Sensor S2 is located outside the region with continuous flames from the propane burner, hence any discrepancies in the modelling of the flame dynamics and resulting burning will directly impact the heat flux predictions at this location. The increase of the convective fluxes due to the arrival of the pyrolysis front at the location of sensor S2 at about $150 \mathrm{~s}$ is only distinctly seen with methods 3 (i.e., 'Nusselt') and 4 (i.e., 'Sgs'), particularly on the finest grid size (i.e., $1 \mathrm{~cm}$ ). Nevertheless, even though the predicted convective heat fluxes with a grid size of $1 \mathrm{~cm}$ are quite similar between methods 3 (i.e., 'Nusselt') and 4 (i.e., 'Sgs'), method 3 does not account for transpiration effects which would significantly reduce the convective heat fluxes due to mass transfer. Ignoring the 'blowing' effect essentially compensates for the lower convective heat fluxes that this method would predict due to the grid dependency on the first cell temperatures.

The differences in the predicted incident radiative heat fluxes remain relatively small between the different methods at sensor S1 (Figure 8), a location which is very close to the propane burner and is constantly engulfed with flames. However, grid sensitivity is observed at sensor S2 (Figure 9), located outside the persistent flame region, with the predictions of method 3 (i.e., 'Nusselt') and method 4 (i.e., 'Sgs') being more sensitive to the choice of the grid size. As expected, higher incident heat fluxes are obtained at both sensor locations on the finest grid (i.e., $1 \mathrm{~cm}$ ) due to sharper flame resolution with higher temperatures and therefore higher total heat fluxes (i.e., convective and radiative), enhancing burning due to the heat feedback mechanism. Focusing on the finest grid size results (i.e., $1 \mathrm{~cm}$ ), it is worth noting that no significant differences are observed in the predicted incident radiative heat fluxes at both sensor S1 (Figure 8) and S2 (Figure 9) during the first $150 \mathrm{~s}$ (i.e., prior to the time of the maximum HRR value). During this initial flame growth period, convective pre-heating of the virgin material is still important since no significant pyrolysis of the combustible walls has occurred yet which would enhance burning and make radiative heat transfer the dominant mode of heat transfer. However, during this period (i.e., first $150 \mathrm{~s}$ ) differences in the predicted convective heat fluxes are present between the various methods (Figures 6-7) which suggests that convection can be important in the initial flame growth period and the subsequent burning behaviour of the flammable walls.

Overall, the fact that the $4 \mathrm{~cm}$ grid size is too coarse can also be observed 


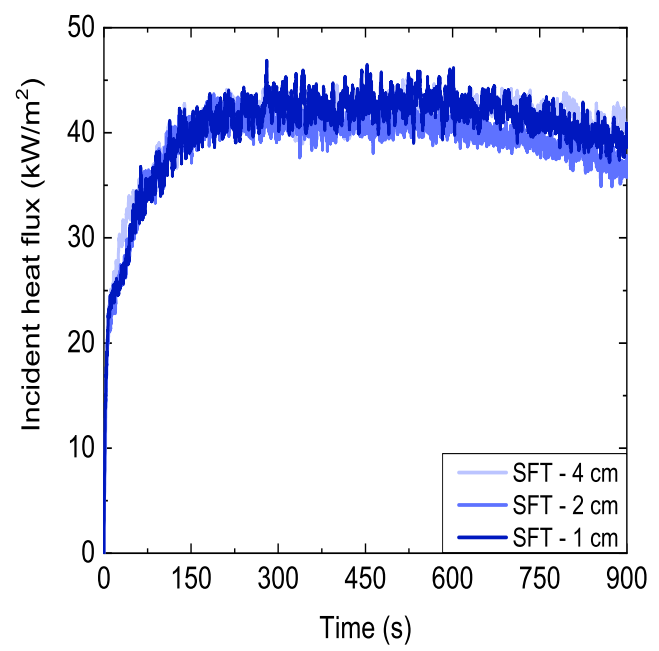

(a)

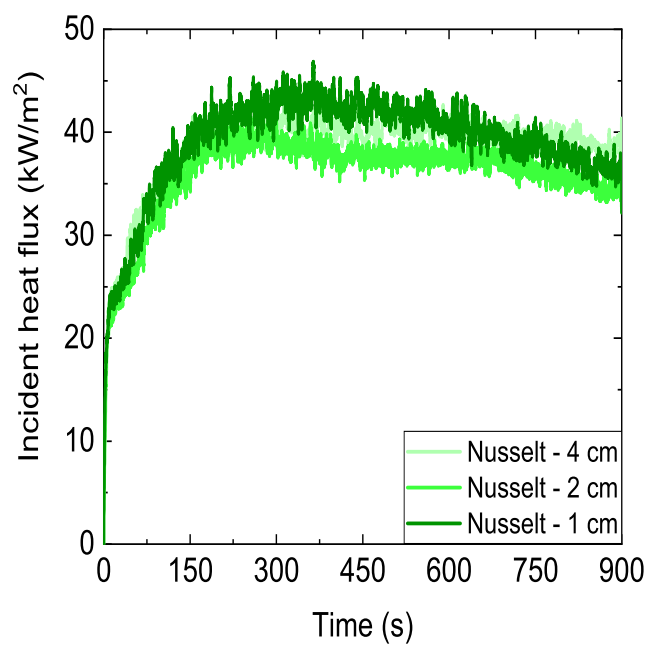

(c)

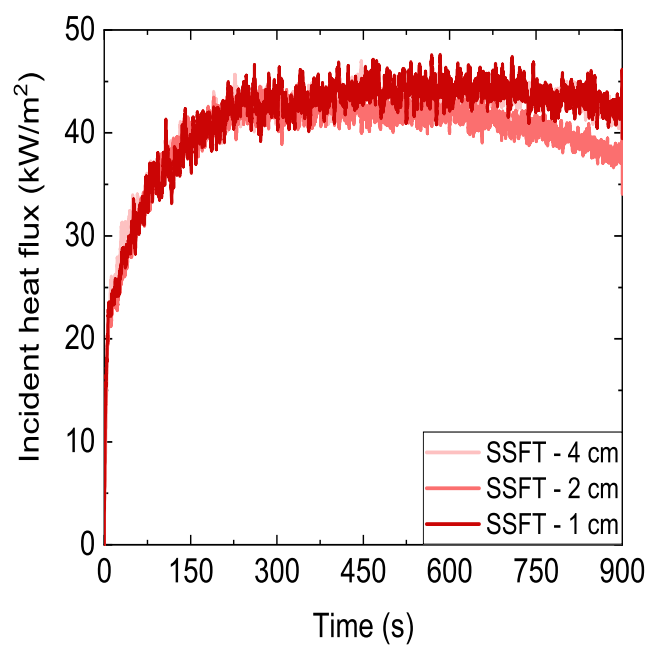

(b)

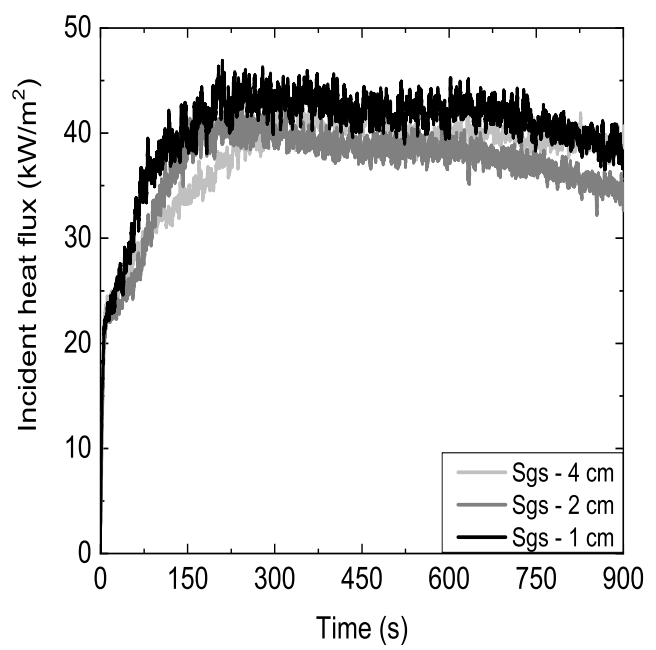

(d)

Figure 8: Temporal evolution of the predicted incident radiative heat fluxes at sensor S1 with method (a) 1-'SFT', (b) 2-'SSFT', (c) 3-'Nusselt' and (d) 4-'Sgs' for the MDF walls as a function of grid size. 


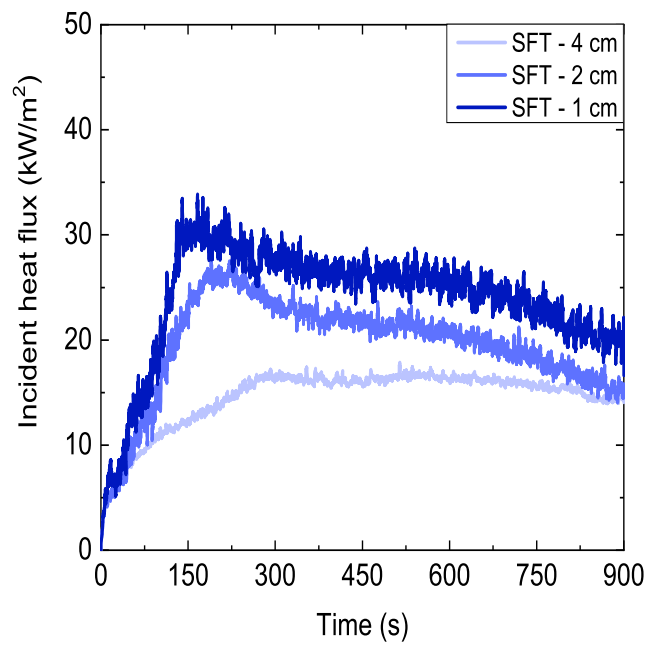

(a)

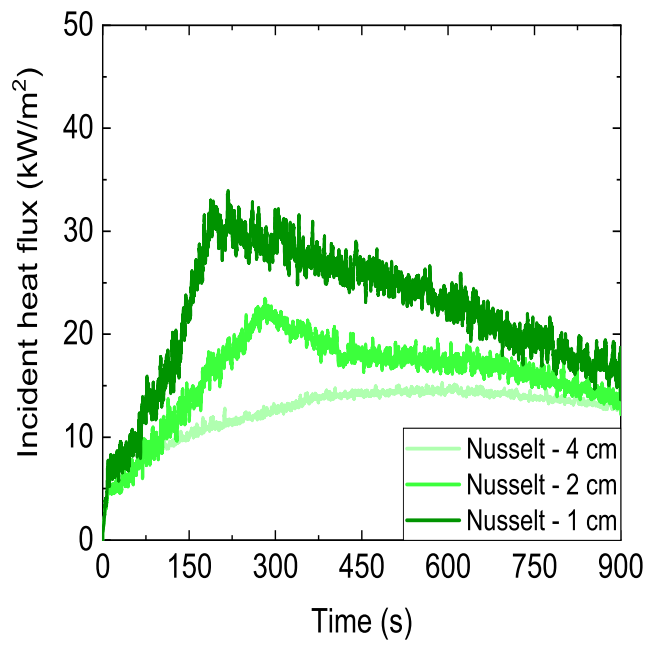

(c)

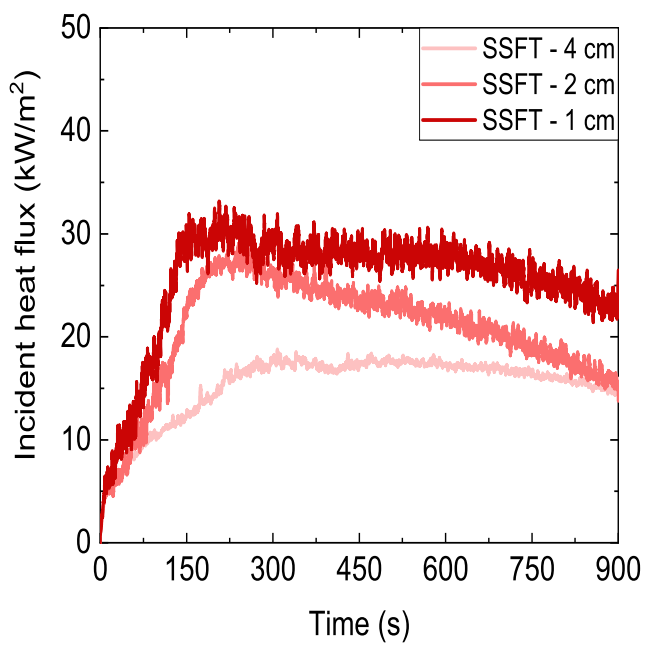

(b)

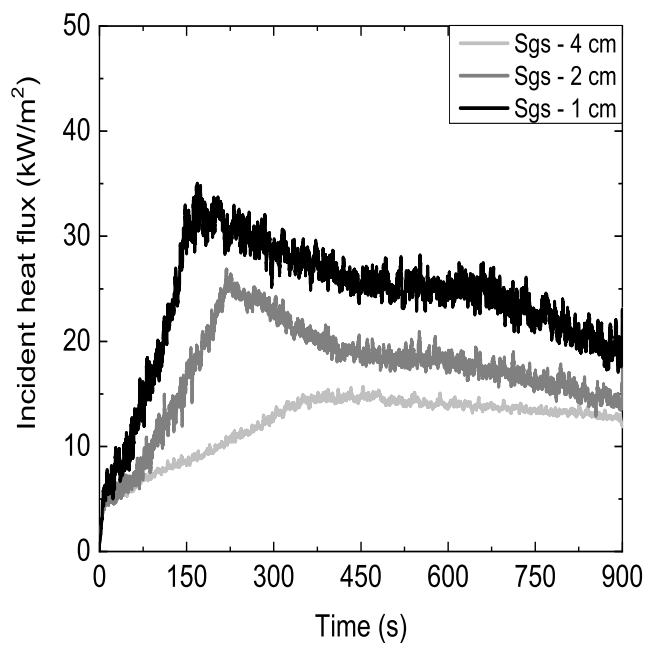

(d)

Figure 9: Temporal evolution of the predicted incident radiative heat fluxes at sensor S2 with method (a) 1-'SFT', (b) 2-'SSFT', (c) 3-'Nusselt' and (d) 4-'Sgs' for the MDF walls as a function of grid size. 
by the lack of significant fluctuations in the convective heat fluxes, compared to the predictions using finer grid sizes, at sensor S1 (Figure 6). The use of coarse grid sizes, combined with numerical diffusion from the discretization schemes, will typically result in an under-estimation of the mixing and the resulting flame temperatures. This aspect is particularly important because it can result in quasi-laminar flow conditions which can greatly affect the heat transfer predictions and the resulting flame spread. It is obvious that the grid size can have a significant influence in flame spread simulations where accurate modelling of (convective and radiative) heat transfer is essential. The latter is a rather important modelling aspect, that needs to be accu-

rately predicted, as radiation is the dominant mode of heat transfer with a significant contribution in the total heat fluxes on the walls. Nevertheless, the former can also have a significant impact on the initial flame growth period when the heating up of the virgin material occurs. The rather grid insensitive predictions of the convective heat fluxes observed with method 1 (i.e., 'SFT') and method 2 (i.e., 'SSFT') on the coarsest grid size (i.e., 4 $\mathrm{cm}$ ) employed in the simulations suggests that the grid size is too coarse for reliable flame spread modelling due to inaccurate prediction of the incident radiative heat fluxes.

\subsection{Total heat fluxes}

The heat fluxes were recorded every $0.1 \mathrm{~s}$ in the simulations and the results are presented by considering a moving average of 20 subsequent time steps (i.e., $2 \mathrm{~s}$ ). It is worth noting that even though the repeatability of the experiments was fairly good in terms of the total HRR, some variation in the total heat fluxes at all three sensor locations examined is present. This aspect also gives an indication of the experimental uncertainty present in the measurements.

The discussion starts with the comparison between the experiments and the numerical predictions for the MDF walls, presented in Figure 10. At first sight, the predicted total heat fluxes with all methods follow the same trends overall. The slightly higher heat fluxes predicted with method 4 (i.e., 'Sgs') at all sensors during the initial flame growth period (i.e., the first $150 \mathrm{~s})$ are consistent with the higher peak HRR obtained with this method. The overestimated HRR values in the decay phase obtained with method 2 (i.e., 'SSFT'), previously presented in Figure 4(a), are reflected here as well with higher total heat fluxes, compared to the other methods, obtained starting from $450 \mathrm{~s}$ onwards. After the peak HRR is reached (i.e., at 150 
s), all methods predict a relatively constant heat flux of $55-60 \mathrm{~kW}$ at sensor S1 (Figure 10(c)). In this case, all simulation results follow closely the experimental data of Experiment 3. The increase in the total heat fluxes, up to approximately $75 \mathrm{~kW} / \mathrm{m}^{2}$, and the subsequent gradual decrease observed experimentally is not fully captured by the simulations at sensor S3 (i.e., Figure 10(a)) with, overall, method 4 (i.e., 'Sgs') performing better. The initial increase and subsequent decrease in the total heat flux profiles (caused by the passing of the flame front) observed experimentally at sensor S2 (i.e., Figure 10(b)) is reasonably well captured by the simulations apart from method 2 (i.e., 'SSFT') which predicts a slower decay in the total heat fluxes (i.e., more intense burning) towards the end of the tests.

The predicted total heat fluxes at all sensor locations for the case involving the plywood walls are presented along with the experimental data in Figure 11. At the location of sensor S1 (Figure 11(c)), the total heat fluxes are underestimated by nearly $10 \mathrm{~kW} / \mathrm{m}^{2}$ by all the methods, except method 2 (i.e., 'SSFT') that predicts heat fluxes approaching the experimental values during the final $300 \mathrm{~s}$. At the location of sensor S2 (Figure 11(b)), the initial increase and the subsequent decrease in the total heat fluxes (caused by the passing of the flame front) are captured adequately by all the methods, most closely by method 2 (i.e., 'SSFT'). At the location of sensor S3 (Figure 11(a)), slightly higher total heat fluxes are obtained with method 4 (i.e.,'Sgs') during the initial flame growth period, while higher values are predicted with method 2 (i.e., 'SSFT') after the peak HRR and until the end of the test, in line with the HRR results previously reported in Figure 3(b). However, all methods predict heat flux values that are similar to the experimental data for most of the remaining duration of the test.

It is worth noting that there is lack of experimental data for individual heat flux components (i.e., radiative and convective) in flame spread scenarios of this scale in literature due to experimental uncertainties /limitations. This is easily verifiable by looking at the available test cases / experimental data in literature as well as the numerous, already published, numerical studies which do not report on a comparison of the individual heat flux components. In most cases, model 'validation' is made by considering individual heat flux components from wall-resolved simulations, e.g., [3, 14, 4, 49], which is a very good alternative even though it does not necessarily exclude any errors in the obtained solutions (e.g., uncertainties in the combustion / radiation modelling in the near wall region).

In order to provide a more quantitative estimate of the balance between 


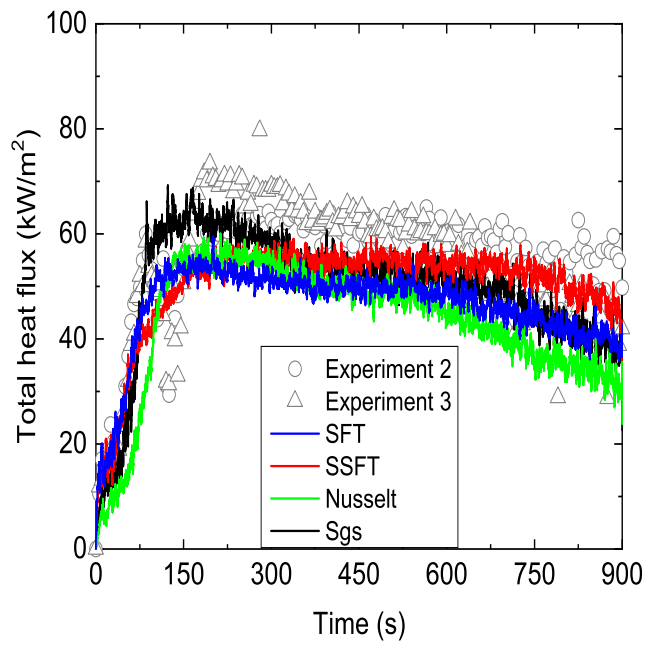

(a)

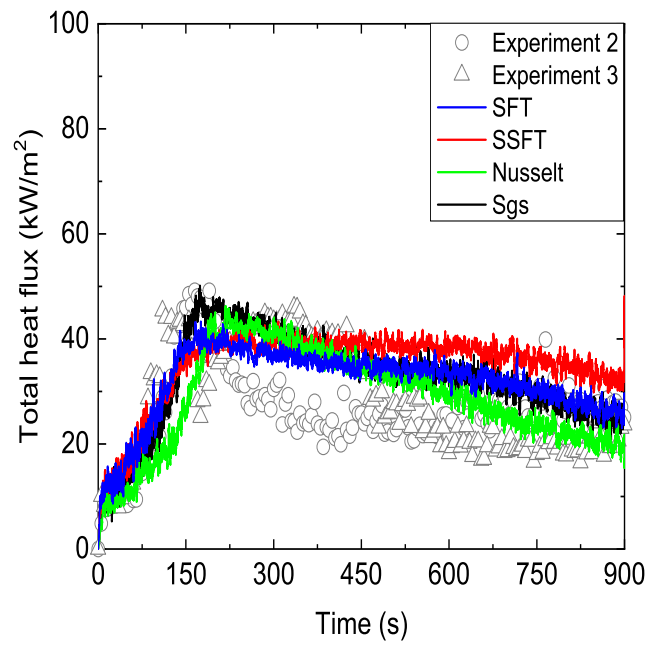

(b)

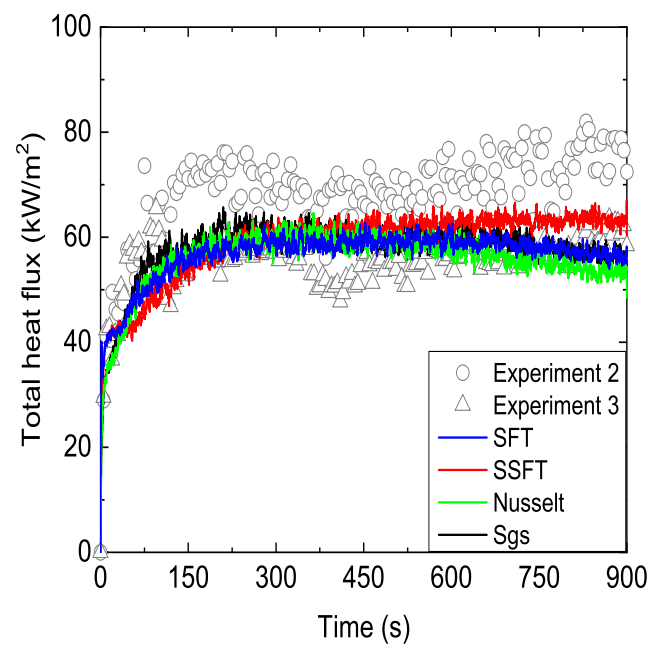

(c)

Figure 10: Temporal evolution of total heat fluxes with MDF at (a) sensor S3, (b) sensor S2 and (c) sensor S1. Methods: 1 -'SFT', 2 -'SSFT', 3 -'Nusselt', 4 -'Sgs'. 


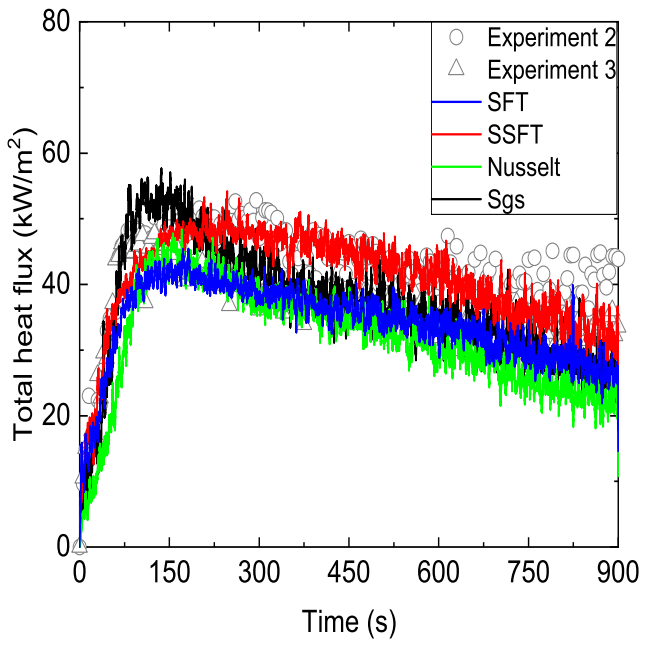

(a)

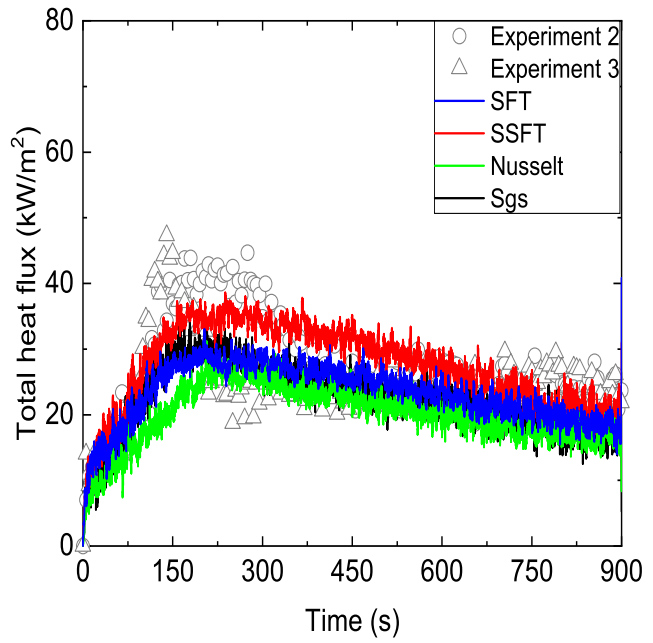

(b)

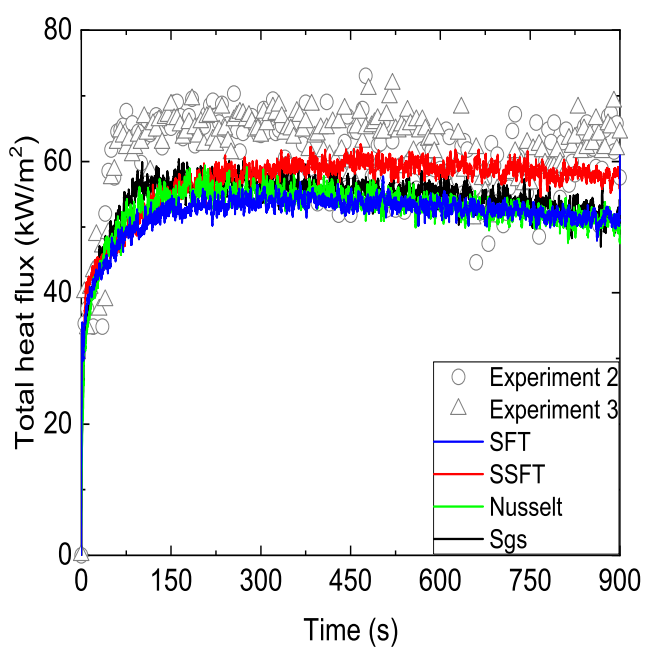

(c)

Figure 11: Temporal evolution of total heat fluxes with plywood at (a) sensor S3, (b) sensor S2 and (c) sensor S1. Methods: 1 -'SFT', 2 -'SSFT', 3 -'Nusselt', 4 -'Sgs'. 
Table 3: Heat feedback to the MDF wall surface with the different methods for convective heat transfer modelling for a grid size of $1 \mathrm{~cm}$.

\begin{tabular}{lccc}
\hline Time & $\begin{array}{c}10 \mathrm{sec} \\
\text { Initial period }\end{array}$ & $\begin{array}{c}155 \mathrm{~s} \\
\text { Maximum HRR }\end{array}$ & $\begin{array}{c}800 \mathrm{~s} \\
\text { Decay phase }\end{array}$ \\
\hline Method 1 ''SFT' & & & \\
Radiative & $33 \%$ & $80 \%$ & $79 \%$ \\
Convective & $67 \%$ & $20 \%$ & $21 \%$ \\
\hline Method 2 ('SSFT') & & & \\
Radiative & $38 \%$ & $81 \%$ & $75 \%$ \\
Convective & $62 \%$ & $19 \%$ & $25 \%$ \\
\hline Method 3 ('Nusselt') & & & \\
Radiative & $65 \%$ & $79 \%$ & $84 \%$ \\
Convective & $35 \%$ & $21 \%$ & $16 \%$ \\
\hline Method 4 ('Sgs') & & & \\
Radiative & $53 \%$ & $76 \%$ & $81 \%$ \\
Convective & $47 \%$ & $24 \%$ & $19 \%$ \\
\hline
\end{tabular}

radiation and convection in the simulations, the heat feedback at the MDF walls at different characteristic times using the different methods for convective heat transfer modelling is presented in Table 3. There are differences in the predicted heat feedback in the initial stages of the tests between the different methods with the method 'SFT' having the highest percentage (i.e., 67\%) compared to $35 \%$ with 'Nusselt' which is the lowest among all methods. The outcome further confirms that convective heating when using the 'Nusselt' method is indeed lower compared to the other methods, hence the differences in the predicted heat release rates previously seen in Figure 4. Once burning of the MDF walls has been established (i.e., after approximately 1.5 minutes), the radiative heat feedback predicted with all convective heat transfer methods varies between $76-84 \%$ throughout the duration of the tests. Overall, the predictions for the heat feedback from the simulations are in reasonably good agreement with [50] where it was reported that, during steady burning, flame radiation accounted for 75 to $80 \%$ of the total of flame radiation plus convection heat transfer in upward turbulent fire spread scenarios (i.e., over vertical PMM slabs).

It is worth noting that the use of the constant radiative fraction model 
in the simulations guarantees that the correct amount of heat will be released due to radiation (i.e., no prediction of the radiative fraction has been attempted in this work). Additionally, the majority of the convective heat transfer methods presented in the paper have already been applied to pool fire scenarios where comparisons to experimental data for the individual heat flux components at the fuel surface were available [51]. In these cases, both the convective and radiative heat fluxes were, in most cases, fairly well predicted which further strengthens the confidence in the numerical modelling presented in this case. These aspects, together with the previously presented results for the heat feedback, even though they do not explicitly validate the convective heat transfer models presented in the paper, suggest that (convective and radiative) heat transfer is fairly well modelled in this work. Nevertheless, further validation to experimental data for individual heat flux components in this case, when they become available, would be the next necessary step.

\subsection{Solid wall temperatures}

A comparison between the predicted wall temperatures from the simulations and the experiments is presented in this section (Figure 12). Results are only presented for the MDF walls, taken as a representative scenario, in order to avoid repetition in the presented results and analysis. Given the uncertainties associated with measuring temperatures near the exposed surface of the flammable walls, emphasis is mainly given on the measured backside temperatures at 6 different locations on the long wall (i.e., positions T1-T6 previously shown in Figure 1). Locations T1-T3 are located close to the propane burner (i.e., $0.2 \mathrm{~m}$ from the bottom of the wall) while positions T4-T6 are located further downstream at a height of $1.4 \mathrm{~m}$ away from the bottom of the long wall.

Even though the simulations follow the experimental trends, the overall temporal evolution of the backside temperatures is not well predicted by the numerical simulations, regardless of the method employed for modelling convective heat transfer. Apart from location T3 (i.e., located near the bottom of the long wall and away from the corner), where there is reasonable agreement with the experimental data, the backside wall temperatures at the other locations are overestimated starting from 300-450 s onward and until the end of the experiments. The discrepancies in the predicted temperatures are present regardless of the method used to model convective heat trans-

fer at the wall surface. Issues related to heat transfer by conduction inside 


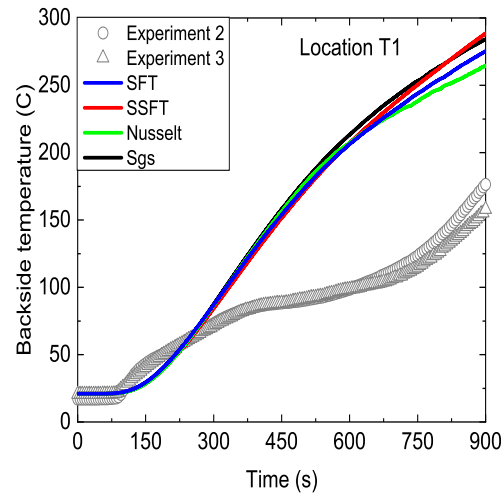

(a)

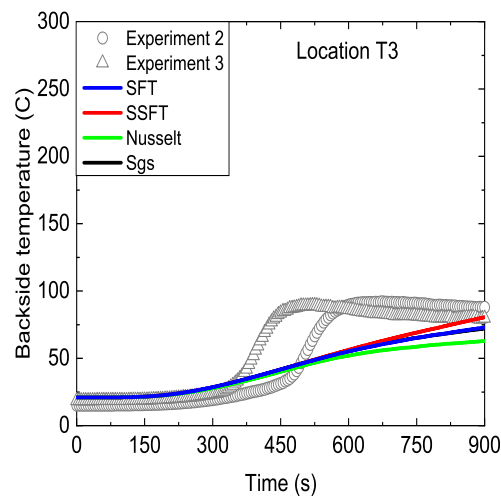

(c)

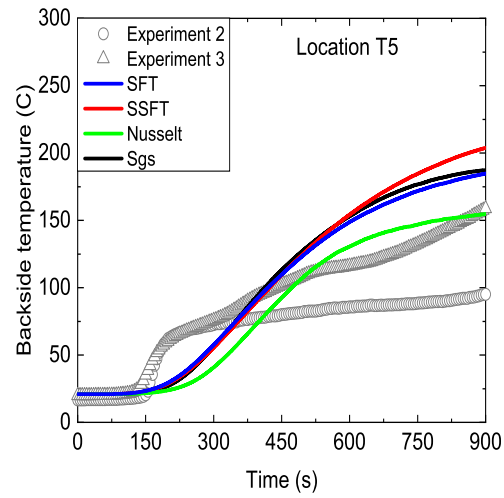

(e)

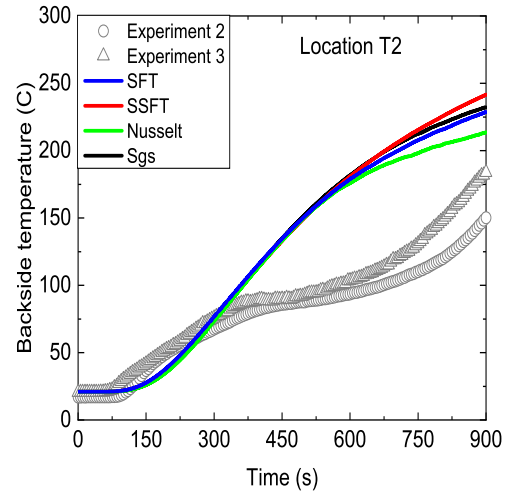

(b)

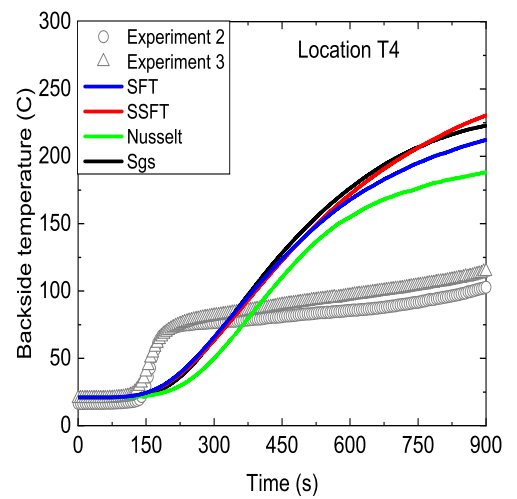

(d)

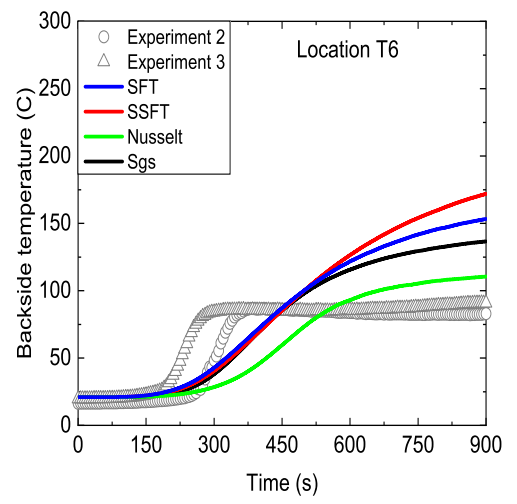

(f)

Figure 12: Temporal evolution of backside temperatures with MDF at location (a) T1, (b) T2, (c) T3, (d) T4, (e) T5 and (f) T6. Methods: 1 -'SFT', 2 -'SSFT', 3 -'Nusselt', 4 -'Sgs'. 
the walls (i.e., linked to the material properties) and the calculation of the heat losses at the back side of the walls could have an influence on this aspect. Moreover, water evaporation and migration of moisture have not been considered in the modelling and the model-effective material properties of the walls (e.g., conductivity, heat capacity, emissivity) are not temperaturedependent. Additionally, a single chemical reaction has been employed for modelling the complex reaction mechanism of wood decomposition. All these modelling limitations contribute to the discrepancies observed between the numerical predictions and the experiments. What is noteworthy, though, is the direct comparison of the CFD results to each other. The lowest backside temperatures are obtained with method 3, and this is in line with the lower total heat fluxes at all sensors from $450 \mathrm{~s}$ onward with this method (Figure 10). Likewise, the overall highest values are obtained with method 2. This is not surprising, given that the processes in the solid phase are modelled in an identical manner, so that the incoming net heat flux at the exposed surface determines what happens at the back side. Overall, improvements in the predictions of the wall temperatures are expected through the use of more advanced modelling approaches related to condensed-phase pyrolysis (i.e., multiple solid reactions, temperature-dependent properties, water migration through the thickness, etc.), consideration of which requires new code implementations in the CFD software employed and is a topic for future work.

\section{Conclusions}

Large eddy simulations of upward flame spread in a corner configuration have been performed focusing on different methods for convective heat transfer modelling. Experiments with MDF and plywood walls have been considered for evaluation purposes, considering a set of Single Burning Item (SBI) tests [20]. Four different methods for modelling the convective heat fluxes at the wall surface were considered: an approach based on the stagnant film theory (method 1), a simplified approach based on the stagnant film theory (method 2), an approach based on Nusselt correlations for natural convection (method 3), and an approach based on the effective (i.e., laminar plus sub-grid scale) thermal diffusivity (method 4). A main difference between methods 1 and 2 versus methods 3 and 4 is that the latter consider temperature directly in the determination of the convective heat fluxes while the former only indirectly through the temperature dependency 
of pyrolysis. Given the grid size employed in the simulations, all approaches aimed at modelling convective heat transfer (i.e., the boundary layer has not effectively been resolved: a $1 \mathrm{~cm}$ mesh was used, whereas a millimeter-sized mesh would be needed for full resolution).

Given the uncertainties related to modelling of such complex scenarios, the initial flame growth period, the peak HRR as well as the decay phase were all fairly well predicted with method 4 for both the MDF and plywood cases. The predictions with method 1 were reasonable in the initial flame growth period but underestimated the peak HRR and overestimated the burning in the decay phase. These discrepancies were linked to the estimation of the convection coefficient which did not properly account for the local properties, and this is a research area that can be further pursued in the future. On the other hand, method 3 predicted the decay phase well but underestimated both the peak HRR and the initial flame growth period, with these discrepancies linked to the temperature sensitivity in the determination of the convection coefficient. Nevertheless, method 3 essentially neglected the blowing effect, which would significantly reduce the convective heat fluxes on the fuel surface and would result in lower predictions for the HRR. This aspect requires further attention in the future, as well as determining whether the use of correlation derived for non-reacting heat transfer problems are applicable to reacting cases involving mass transfer. Finally, method 2 predicted the initial flame growth period fairly well but underestimated the peak HRR and did poorly in the decay phase by overestimating the burning of the walls in both cases. These discrepancies were linked to the fact that the local flow properties are not accounted for by method 2, with the convective heat fluxes only changing in the presence of mass transfer. Even though the predicted total heat fluxes with all methods followed the same trends, subtle differences among them in the initial flame growth period, a period which is important for the pre-heating of the virgin material due to convection, were evident which affected the predicted HRR as well. Overall, the predicted total heat fluxes with method 4 were closer to the experimental data. This is while the temporal evolution of the backside temperatures on several locations on the flammable walls were not well captured by the numerical simulations with either method.

The influence of grid size was shown to be important for the predicted incident heat fluxes on the flammable walls, both radiative and convective. Specifically, methods 3 and 4 exhibit more mesh sensitivity than methods 1 and 2 , because the latter methods do not use the local temperatures (in the 
first grid cell) for the calculation of the convective heat fluxes. This makes the calculations somewhat more robust but at the cost of not considering the local temperature directly (it is considered only indirectly, through the temperature dependency of pyrolysis). It is also noted that mesh sensitivity in the radiative heat fluxes is observed for all four methods indicating that, even though accurate modelling and prediction of convective heat transfer can be important, the effect of radiative heat transfer is still dominant in flame spread scenarios.

Overall, it was demonstrated that the influence of convective heat transfer modelling is important in scenarios involving upward flame spread. Failing to accurately model convection, which can significantly contribute in the pre-heating of the virgin material during the early stages of flame spread, can result in slower flame growth, which subsequently influences the burning behaviour (i.e., time to peak HRR, peak HRR value, decay phase) of the flammable walls in general.

\section{Acknowledgments}

This research has been funded by Ghent University (Belgium) through GOA project BOF16/GOA/004.

\section{References}

[1] S.R. Tieszen, On the fluid mechanics of fires, Annu. Rev. Fluid Mech. 33 (2001) 67-92.

[2] P. Joulain, The behavior of pool fires: state of the art and new insights, Proc. Comb. Inst. 27 (1998) 2691-2706.

[3] N. Ren, Y. Wang, S. Vilfayeau, A. Trouvé, Large eddy simulation of turbulent vertical wall fires supplied with gaseous fuel through porous burners, Combust. Flame 169 (2016) 194-208.

[4] N. Ren, Y. Wang, A Convective Heat Transfer Model for LES Fire Modeling, Proc. Comb. Inst. 38 (2021) 4535-4542.

[5] F.P. Incropera, D.P. DeWitt, T.L. Bergman, A.S. Lavine, Fundamentals of Heat and Mass Transfer, 6th Edition, John Wiley \& Sons, New York, 2006, pp. 571-579. 
[6] Z. Zhai, Q.Y. Chen, Numerical determination and treatment of convective heat transfer coefficient in the coupled building energy and CFD simulation, Build. Environ. 39 (2004) 1001-1009.

[7] https://github.com/fireFoam-dev

[8] Y. Wang, K.V. Meredith, X. Zhou, P. Chatterjee, Y. Xin, M. Chaos, N. Ren, S.B. Dorofeev, Numerical Simulation of Sprinkler Suppression of Rack Storage Fires, Fire Saf. Sci. 11 (2014) 1170-1183.

[9] D. Zeinali, A. Gupta, G. Maragkos, G. Agarwal, T. Beji, M. Chaos, Y. Wang, J. Degroote, B. Merci, Study of the importance of non-uniform mass density in numerical simulations of fire spread over MDF panels in a corner configuration, Combust. Flame 200 (2019) 303-315.

[10] Z. Yan, G. Holmstedt, CFD Simulation of Upward Flame Spread over Fuel Surface, Fire Saf. Sci. 5 (1997) 345-356.

[11] Fire Dynamics Simulator (FDS) available through https://pages.nist.gov/fds-smv/

[12] N. Ren, Y. Wang, S. Vilfayeau, A. Trouvé, Large Eddy Simulation of Propylene Turbulent Vertical Wall Fires, Proceedings of the seventh international seminar on fire and explosion hazards, Providence, RI, USA, 2013.

[13] U. Piomelli, E. Balaras, Wall-layer models for large-eddy simulations, Annu. Rev. Fluid Mech., 34 (2002) 349-374.

[14] N. Ren, Y. Wang, A. Trouvé, Large Eddy Simulation of Vertical Turbulent Wall Fires, Procedia Eng. 62 (2013) 443-452.

[15] K. Fukumoto, C. Wang, J. Wen, Large eddy simulation of upward flame spread on PMMA walls with a fully coupled fluid-solid approach, Combust. Flame 190 (2018) 365-387.

[16] E. Markus, A. Snegirev, E. Kuznetsov, L. Tanklevskiy, Application of the thermal pyrolysis model to predict flame spread over continuous and discrete fire load, Fire Saf. J. 108 (2019) 102825. 
[17] N. Ren, J. de Vries, X. Zhou, M. Chaos, K.V. Meredith, Y. Wang, Largescale fire suppression modeling of corrugated cardboard boxes on wood pallets in rack-storage configurations, Fire Saf. J. 91 (2017) 695-704.

[18] A.V. Singh, M.J. Gollner, A methodology for estimation of local heat fluxes in steady laminar boundary layer diffusion flames, Combust. Flame 162 (2015) 2214-2230.

[19] G. Maragkos, B. Merci, Grid insensitive modelling of convective heat transfer fluxes in CFD simulations of medium-scale pool fires, Fire Saf J. (2020) 103104, In Press.

[20] D. Zeinali, S. Verstockt, T. Beji, G. Maragkos, J. Degroote, B. Merci, Experimental study of corner fires : part II : flame spread over MDF panels, Combust. Flame 189 (2018) 491-505.

[21] D. Zeinali, Flame spread and fire behavior in a corner configuration, PhD thesis, Ghent University, 2019.

[22] G. Maragkos, T. Beji, B. Merci, Advances in modelling in CFD simulations of turbulent gaseous pool fires, Combust. Flame 181 (2017) 22-38.

[23] G. Maragkos, T. Beji, B. Merci, Towards predictive simulations of gaseous pool fires, Proc. Comb. Inst. 37 (2019) 3927-3934.

[24] P. Moin, K. Squires, W.H. Cabot, S. Lee, A dynamic subgrid-scale model for compressible turbulence and scalar transport, Phys. Fluids A 3 (1991) 2746-2757.

[25] G. Maragkos, B. Merci, On the use of dynamic turbulence modelling in fire applications, Combust. Flame 26 (2020) 9-23.

[26] C. Fureby, G. Tabor, Mathematical and Physical Constraints on Large Eddy Simulations, Theoret. Comput. Fluid Dynamics 9 (1997) 85-102.

[27] B.F. Magnussen, B.H. Hjertager, On mathematical modeling of turbulent combustion with special emphasis on soot formation and combustion, Proc. Comb. Inst. 16 (1977) 719-729.

[28] E.A. Brizuela, R.W. Bilger, On the eddy break-up coefficient, Combust. Flame 104 (1996) 208-212. 
[29] A. Hamins, K. Konishi, P. Borthwick, T. Kashiwagi, Global properties of gaseous pool fires, Proc. Comb. Inst. 26 (1996) 1429-1436.

[30] SFPE Handbook of Fire Protection Engineering, Springer, 2016.

[31] M.F. Modest, The Weighted-Sum-of-Gray-Gases Model for Arbitrary Solution Methods in Radiative Transfer, J. Heat Transfer 113 (1991) 650-656.

[32] J.G. Quintiere, Fundamentals of fire phenomena, John Wiley \& Sons, 2006, pp. 248-249.

[33] T. Ahmad, G.M. Faeth, Turbulent wall fires, Proc. Comb. Inst. 17 (1979) 1149-1160.

[34] D. Drysdale, An Introduction to Fire Dynamics, Willey, 2011.

[35] P.L. Blackshear, K.A. Murty, Heat and mass transfer to, from, and within cellulosic solids burning in air, Symposium (International) on Combustion 10 (1965) 911-923.

[36] W.H. McAdams, Heat Transmission, McGraw-Hill Book Company, New York, 1957.

[37] R.J. McDermott, Quality assessment in the fire dynamics simulator: a bridge to reliable simulations, Proceedings of the Fire and Evacuation Modeling Technical Conference, Maryland, USA, August 15-16, 2011.

[38] P.L Betts, I.H Bokhari, Experiments on turbulent natural convection in an enclosed tall cavity, Int. J. Heat Fluid Flow 21 (2000) 675-683.

[39] S. Vilfayeau, Large Eddy Simulation of Fire Extinction Phenomena, PhD Thesis, University of Maryland, USA, 2015.

[40] https://www.openfoam.com/documentation/guides/latest/doc/guideverification-validation.html

[41] P. Renze, K. Akermann, Simulation of Conjugate Heat Transfer in Thermal Processes with Open Source CFD, ChemEngineering 2019, 3, 59.

[42] [L. Hao, Development of a suite of verification tests for the CFD fire model FireFOAM, Master thesis, University of Maryland, USA, 2017. 
[43] M. Chaos, Spectral Aspects of Bench-Scale Flammability Testing: Application to Hardwood Pyrolysis, Fire Saf. Sci. 11 (2014) 165-178.

[44] M. Chaos, M.M. Khan, N. Krishnamoorthy, J.L. de Ris, S.B. Dorofeev, Evaluation of optimization schemes and determination of solid fuel properties for CFD fire models using bench-scale pyrolysis tests, Proc. Combust. Inst. 33 (2011) 2599-2606.

[45] G. Agarwal, M. Chaos, Y. Wang, D. Zeinali, B. Merci, Pyrolysis Model Properties of Engineered Wood Products and Validation Using Transient Heating Scenarios, Interflam, 14th International Conference and Exhibition on Fire Science and Engineering, Nr Windsor, UK, 2016.

[46] A.S. Rangwala, S.G. Buckley, J.L.Torero, Analysis of the constant Bnumber assumption while modeling flame spread, Combust. Flame 152 (2008) 401-414.

[47] M.J. Spearpoint, J.G. Quintiere, Predicting the burning rate of wood using an integral model, Combust. Flame 123 (2000) 308-324.

[48] M. Janssens, Fundamental thermophysical characteristics of wood and their role in enclosure fire growth, PhD thesis, Ghent University, 1991.

[49] K. Li, S. Hostikka, Embedded flame heat flux method for simulation of quasi-steady state vertical flame spread, Fire Saf. J. 104 (2019) 117-129.

[50] L. Orloff, J. De Ris, G.H. Markstein, Upward turbulent fire spread and burning of fuel surface, Symposium (International) on Combustion 15 (1975) 183-192.

[51] G. Maragkos, B. Merci, Grid insensitive modelling of convective heat transfer fluxes in CFD simulations of medium-scale pool fires, Fire Saf. J. (2020) 103104 (In Press, Corrected Proof). 